\title{
Definition of the Caribbean Islands biogeographic region, with checklist and recommendations for standardized common names of amphibians and reptiles
}

\author{
S. Blair Hedges ${ }^{1, *}$, Robert Powell ${ }^{2}$, Robert W. Henderson ${ }^{3}$, Sarah Hanson ${ }^{1}$, and John C. Murphy ${ }^{4}$ \\ 'Center for Biodiversity, Temple University, 1925 N. 12th Street, Philadelphia, Pensylvania 19122, USA. \\ 2Department of Biology, Avila University, 11901 Wornall Road, Kansas City, Missouri 64145, USA. \\ ${ }^{3}$ Vertebrate Zoology, Milwaukee Public Museum, 800 West Wells Street, Milwaukee, Wisconsin 53233, USA. \\ ${ }^{4}$ Science and Education, Field Museum of Natural History, 1400 Lake Shore Drive, Chicago, IL 60616 USA. \\ *Corresponding author (sbh@temple.edu) \\ Edited by: R. Graham Reynolds. Date of publication: 28 May 2019. \\ Citation: Hedges SB, Powell R, Henderson RW, Hanson S, and Murphy JC. 2019. Definition of the Caribbean Islands biogeographic region, with \\ checklist and recommendations for standardized common names of amphibians and reptiles. Caribbean Herpetology, 67, 1-53. \\ DOI: $10.31611 /$ ch.67
}

\begin{abstract}
To facilitate biological study we define "Caribbean Islands" as a biogeographic region that includes the Antilles, the Bahamas, and islands bordering Central and South America separated from mainland areas by at least 20 meters of water depth. The advantages of this definition are that it captures nearly all islands with endemic species and with at least some Antillean-derived species, and still circumscribes a region of high biodiversity and biogeographic significance. We argue that Caribbean islands, in this expanded sense, are also cohesive from a conservation standpoint in that they share high human population densities and similar conservation threats. A disadvantage of this definition, strictly applied, is that it includes some islands (e.g., Trinidad) that have mostly mainland species. However, we propose that researchers can increase the stringency of the definition so that it is less inclusive, and make comparisons between different definitions as needed. We provide an updated checklist with standardized common English names for the 1,013 species of amphibians and reptiles occurring in the region, along with principles for constructing common names.
\end{abstract}

Keywords: Caribbean Islands, West Indies, Antilles, sea level, dispersal, biogeography, biota, vertebrates.

\section{A biological definition of "Caribbean Islands"}

The biota of the greater Caribbean region is one of the most intensely studied in the world, especially because of its diversity and high levels of endemism. It is a complex region in many respects, including geology and biogeography. This complexity has led to varying geographic definitions of the region, leading in turn to considerable potential for confusion. In this article we provide an expanded definition of the Caribbean islands biogeographic region to improve the quality and comparability of research on biodiversity and conservation. We also provide an updated checklist of the amphibians and reptiles, the two major vertebrate groups inhabiting Caribbean islands, along with standardized English common names.

Most biological studies of Caribbean terrestrial biota refer to a region called the "West Indies," a name that traces its origins to the time of European discovery. The geographical definition of the West Indies has varied over time (e.g., Hedges 2018a, Hopkins 1997) but is frequently defined as including the Greater Antilles, the Lesser Antilles, the Bahamas, and the islands of the southern Caribbean (Southern Antilles) off the northern coast of 
Venezuela (ABC Islands, Trinidad and Tobago, Isla Margarita, etc.). However, most biologists define the West Indies differently, omitting the islands of the southern Caribbean and tailoring the definition in other ways. For example, an ornithologist (Bond 1960) added the Swan Islands, San Andres, and Providencia whereas herpetologists have either added those three islands (e.g., Schwartz and Thomas 1975; Schwartz and Henderson 1991) or only the Swan Islands (e.g., Powell and Henderson 2012). A review of bats (Morgan 2001) omitted Grenada, The Grenadines, and San Andres from the definition of the West Indies but included Providencia. Schwartz and Thomas (1975) admitted "Some arbitrariness in defining the West Indies."

One alternative has been to characterize the region as "Caribbean Islands." For example, the Caribbean Islands Hot Spot (Smith et al. 2005) includes the primary West Indian islands (Greater Antilles, Lesser Antilles, and Bahamas) as well as the Swan Islands, and some but not all of the islands of the southern Caribbean, omitting Trinidad, Tobago, Los Testigos, Isla Margarita, and Isla La Tortuga. One major problem with the name "Caribbean Islands" is that the Bahamas and the Turks and Caicos are not in the Caribbean Sea, but rather in the Atlantic Ocean. On the other hand, the northern and eastern borders of the Caribbean Sea, where it contacts the Atlantic Ocean, are somewhat arbitrary, defined by references to lighthouses, coordinates, and water depths.

The International Hydrography Organization $(\mathrm{IHO})$, the authority charged with surveying and charting oceans and seas, defines the Caribbean Sea as follows: "IN The Yucatan Channel. A line joining Cape Catoche Light $\left(21^{\circ} 37^{\prime} \mathrm{N}, 87^{\circ} 04^{\prime} \mathrm{W}\right)$ with the Light on Cape San Antonio in Cuba. ON THE NoRTH. In the Windward Channel: a line joining Caleta Point $\left(74^{\circ} 15^{\prime} \mathrm{W}\right)$ and Pearl Point $\left(19^{\circ} 40^{\prime} \mathrm{N}\right)$ in Haiti. In the Mona Passage: a line joining Cape Engano and the extreme of Agujereada $\left(18^{\circ} 31^{\prime} \mathrm{N}, 67^{\circ} 08^{\prime} \mathrm{W}\right)$ in Puerto Rico. EAstern Limits. From Point San Diego (Puerto Rico) northward along the meridian thereof $\left(65^{\circ} 39^{\prime} \mathrm{W}\right)$ to the 100 fathom line, thence eastward and southward, in such a manner that all islands, shoals and narrow waters of the Lesser Antilles are included in the Caribbean Sea as far as Galera Point (Northeast extremity of the island of Trinidad). From Galera Point through Trinidad to Galeota Point (southeast extreme) and thence to Baja Point $\left(9^{\circ} 32^{\prime} \mathrm{N}, 61^{\circ} \mathrm{W}\right)$ in Venezuela" (IHO 1953).

However, the Bahamas and the Turks \& Caicos have long been culturally and politically associated with Caribbean islands. In addition to the historical connection with the West Indies, The Bahamas and Turks and Caicos are members of the Caribbean Community (CARICOM 2018), an organization of Caribbean nations and dependencies established to promote economic integration and cooperation among its members, much like the European Union. In essence, the Bahamas are Caribbean islands in the Atlantic Ocean. To bring the geographical definition more in line with modern usage, the IHO probably should extend the northern border of the Caribbean Sea to include the islands of the Bahamas and the Turks \& Caicos.

Irrespective of the definition of the Caribbean Sea, we believe that the "Caribbean islands" should include the Bahamas and the Turks \& Caicos, in addition to the Greater and Lesser Antilles, because they all form a cohesive core-Antillean biota (e.g., Hedges 2006). The Bahamas and the Turks \& Caicos are low islands on banks that were flooded as recently as the Pleistocene, with their vertebrate fauna being derived almost entirely by dispersal from the Greater Antilles (e.g., Hedges 1996). However, on islands bordering the mainland in the western and southern Caribbean, the percentage of core-Antillean elements is lower and mainland-derived species higher. This is the primary reason why past studies and checklists of Caribbean islands, excluded or only partly included these islands. As expected, islands closest to the mainland, such as Trinidad (Murphy et al. 2018), have a higher percentage of mainland species than islands farther from the mainland, such as the Swan islands (McCranie et al. 2017), raising the question as to where to draw the line.

Conservation is another factor we considered in redefining "Caribbean Islands." Islands in general have more fragile ecosystems than mainland areas, resulting in greater conservation threats to island species (IUCN 2018). First, because islands often have fewer taxonomic groups than mainland areas (e.g., Williams 1989), introduced species are less likely to be outcompeted and more likely to survive, negatively impacting the native species (e.g., Simberloff 1995). Secondly, islands have denser human populations and correspondingly suffer greater habitat disturbances than mainland areas (FAO 2015; United Nations 2018). Thirdly, population sizes of endemic 
species are smaller (albeit often denser) on islands, largely because of limited space, and therefore extirpations of habitats and populations have a greater impact on species survival.

For these reasons, we advocate recognition of all Caribbean islands, including the Bahamas and the Turks \& Caicos, as part of a single and cohesive region of significance for biogeography, biodiversity, and conservation. However, we use a depth cutoff of 20 meters (the shallowest underwater connection to the mainland) to exclude the many ephemeral barrier islands and other islands only recently established along the Caribbean coast of Central and South America. In essence, this definition is evolution-based, favoring islands more likely to have endemics and a longer history of isolation, and therefore of greater interest to those studying biodiversity and conservation. Researchers may choose to use the entire region as defined (Figs. 1-3) or employ a stricter definition as needed. For example, stricter definitions could include a deeper connector-depth cutoff, a larger minimum island size, or selection of islands with only a high proportion of endemic species.

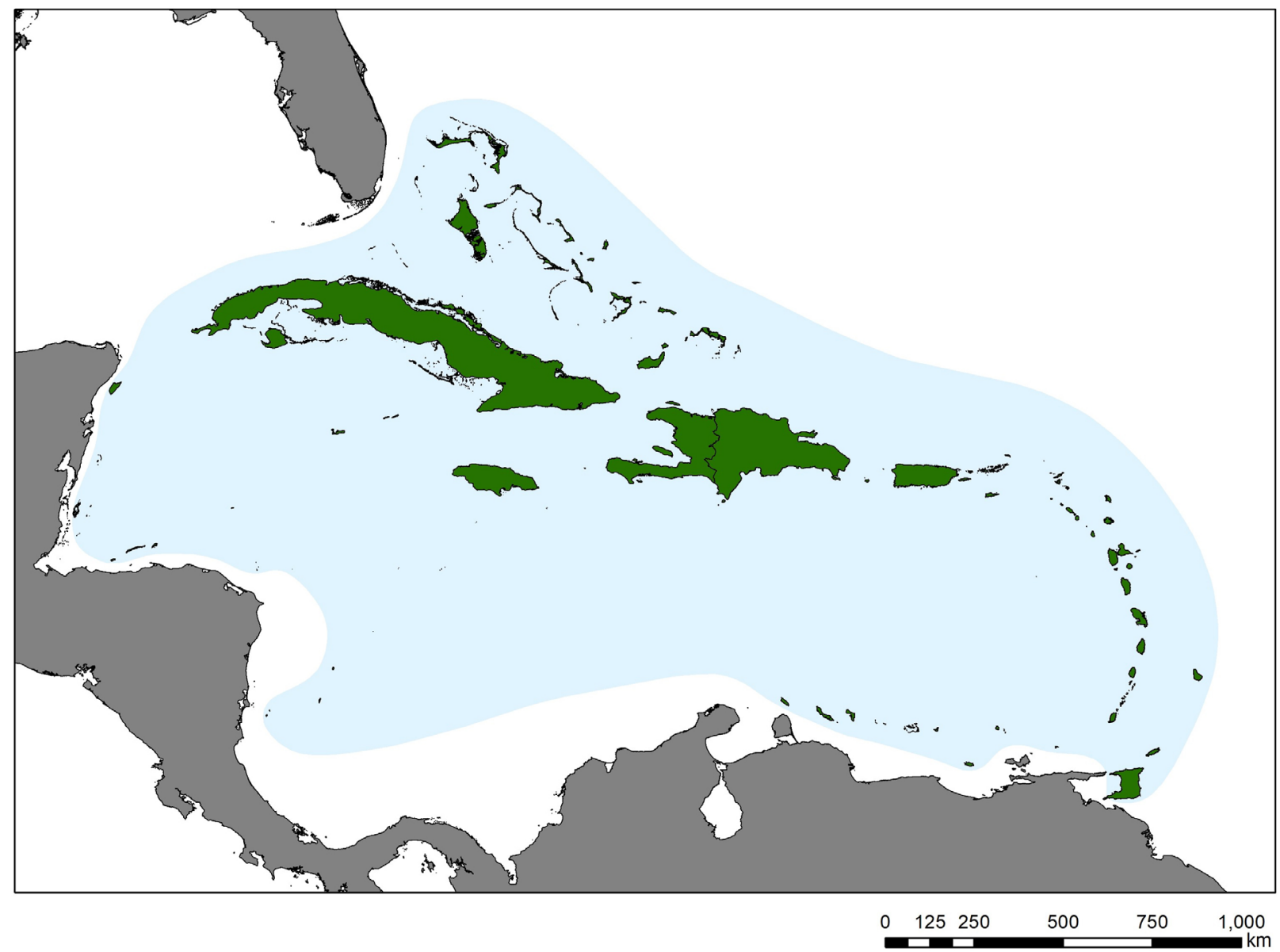

Figure 1. The region herein defined as "Caribbean Islands." The pale blue shading includes islands separated from the mainland by at least 20 meters of water depth. 
Because the sea level was 20 meters lower approximately 10,000 years ago (Khan et al. 2017), islands defined using this connector depth have been isolated from the mainland for that period, not considering local tectonic effects on sea level. Earlier periods of high sea level, such as 3.2-3.0 million years ago, 424-395,000 years ago, and 129,000-116,000 years ago (Miller et al. 2012; Dutton et al. 2015) also could have isolated populations. Colder periods, including glacial maxima, would have exposed land, allowing dispersal and potential gene flow. However, those low-elevation areas likely would have been drier and less hospitable as habitat, which would have limited dispersal. Therefore, the history of isolation of lineages and speciation could be complex and not strictly determined by marine barriers.

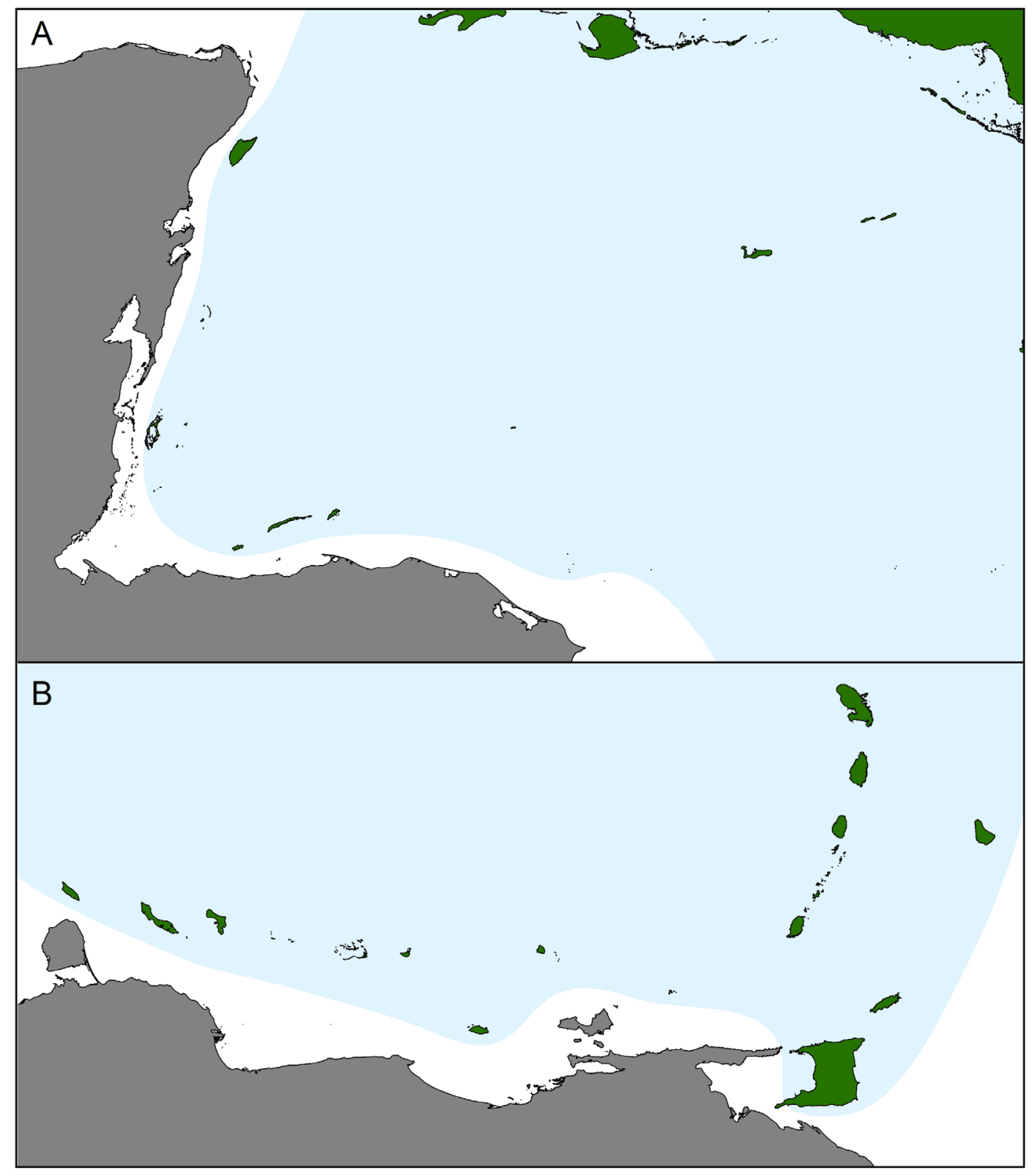

Figure 2. Closeups of two regions in Figure 1 showing details of the western and southeastern region. 
Amphibians and reptiles occur on at least 909 Caribbean Islands and include 1,013 currently recognized species (van Buurt 2004; McCranie et al. 2005; Powell and Henderson 2012; Hedges 2018b; Murphy et al. 2018). In the checklist below, we list each of those species along with standardized English common names. One of us (SBH) has maintained such a checklist for nearly two decades in the database Caribherp (Hedges 2018b). However, the list here is for the more inclusive region defined here (Caribbean Islands), and is formalized and annotated. In addition, we present a set of guidelines for creating standardized common names, which are applicable to other regions of the world and other groups of organisms.

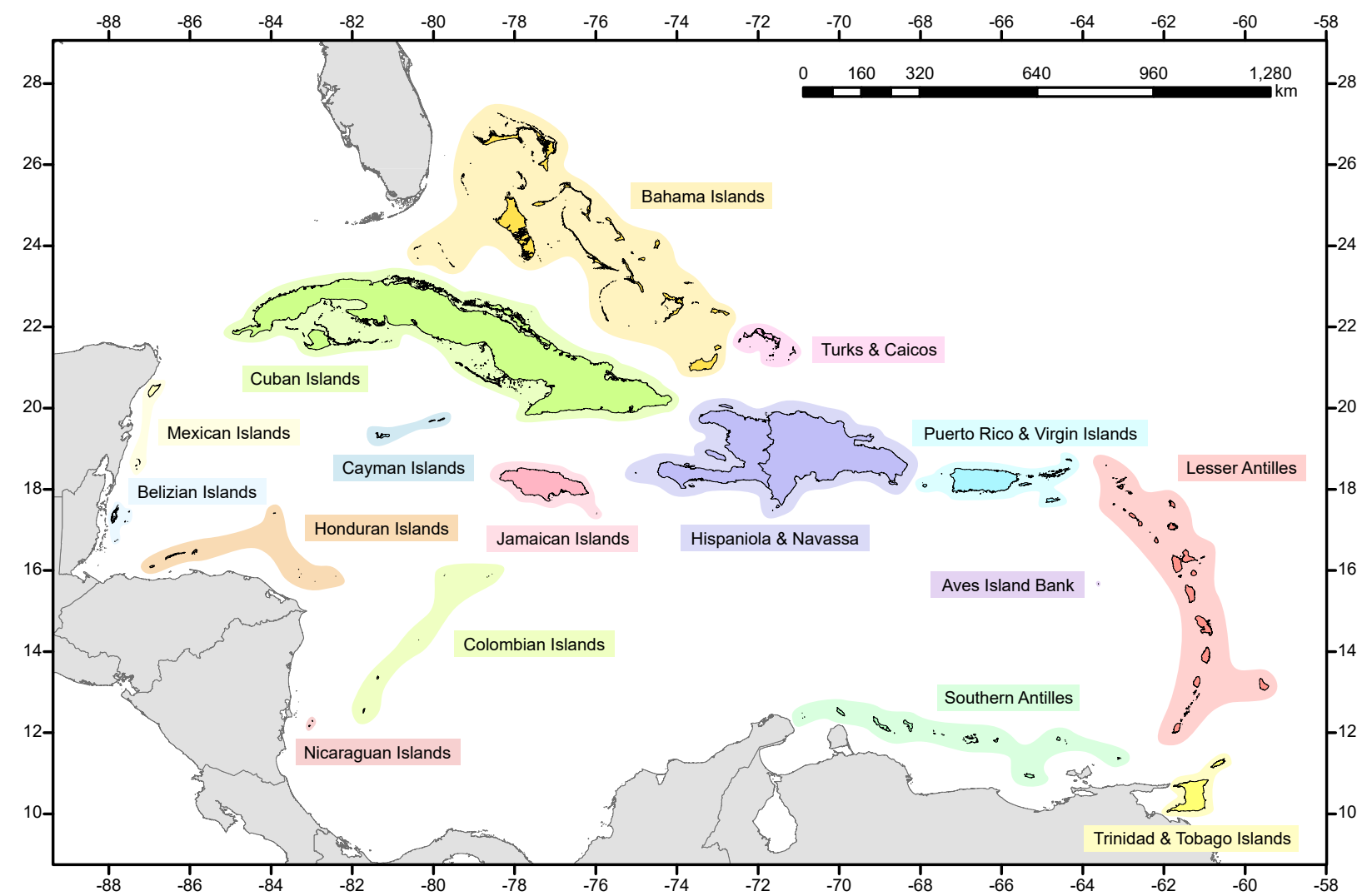

Figure 3. Caribbean islands, as defined herein, labelled according to island groups.

\section{Standardized English Common Names for Caribbean Amphibians and Reptiles}

Our goal was to generate a list of English common names for all species of amphibians and reptiles on Caribbean islands, but also with the further intent of this list eventually being part of a Western Hemisphere-wide or even a global list of standardized English names. In the process, we have assembled our own guidelines for common name creation, pulling together what we consider the best practices of previous authors combined with some new and modified criteria.

Rather than a list of rules, our guidelines consist of three principles that we consider fundamental to the creation of common names: uniqueness, usefulness, and consistency. Together, we believe that following these three principles will lead to an ideal and stable system of names, much like the list of Sibley \& Monroe (1990) for birds.

Principle 1. Uniqueness. The ideal name should be unique in the world or at the very least in a major part of the world (i.e., the Americas). This is important because it avoids overlap and confusion with species in other regions, 
which is a problem in many regional field guides and lists, such as that for North America (Crother 2012). Having different sets of standards for North, Central, and South America and the Caribbean region serves no one well and leads to such names as "Green Anole" for a single North American species when, in fact, many green anoles occur throughout the Western Hemisphere. The same is true for other names in the North American list, including, for

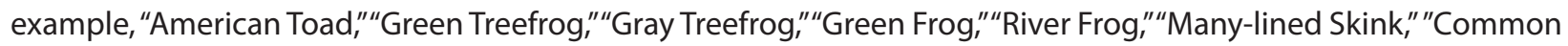
Wormsnake," and "Common Watersnake."

Unique names can be created quickly by using patronyms (either the dedicated Latin species name or author of the Latin species name) or a reference to the type locality. This was done in the North American list (e.g., "Cope's Gray Treefrog," "Kirtland's Snake," "Blanding's Turtle"), the Mexican list (Liner 1994), to some degree in the checklist to the world's turtles (Turtle Taxonomy Working Group 2017), but most extensively in an ambitious attempt to generate a global list (Frank and Ramus 1995). However, a patronym does not convey information about the animal itself, such as appearance or geographic distribution. Likewise, the type locality of a species, especially if it occurs in other locations (which is common), has little diagnostic value. Therefore, we do not recommend either option. Similarly, we disagree with the rule in the list of standardized English names of North American amphibians and reptiles (Crother 2012) that "long-established names in widespread use should be retained, regardless of any inaccuracy of description, behavior, habitat, location or phylogenetic relationship suggested by the name, unless there is a compelling and special reason." Tradition should not be favored over accuracy. Unlike established scientific names, common names can change and should be accurate. Instead, we recommend that unique names should also be useful names, which is our second principle.

Principle 2. Usefulness. As a rule complementary to the previous one, we mean that an ideal name should provide useful information facilitating the identification of the species. For example, we could have named Sphaerodactylus oxyrhinus Gosse 1850 as "Gosse's Gecko" but that would not have conveyed any useful information about the animal or where it occurs. We instead named it the "Jamaican Sharp-nosed Geckolet," which describes it as a small gecko from Jamaica with a pointed nose. We avoided excessively lengthy names with more than three or four words by compounding and adding standardized suffixes (e.g., "geckolet" for a small gecko).

Colloquial names, in English or other languages, such as Zandolie (used for Ameiva fuscata on Dominica, A. atrigularis in Trinidad, and for most lizards in Haiti), usually violate guidelines designed for use across a wider region and we therefore avoided them in this list. Also, colloquial names are frequently in a language other than English and therefore the English-language meaning could be lost.

Principle 3. Consistency. Names should conform to the standard rules of English grammar. For consistency, we use American English but recognize that British and other English dialects are equally acceptable in all instances.

Standardized English names of species should be capitalized to distinguish them from descriptions and generalized usage.-When group names (i.e., standardized English names for genera and higher categories or as a word or words that applies to one or more species) are used alone (i.e., not as part of the English name of a species), they should not be capitalized unless they are proper nouns. For example, "The Cuban Green Anole is a commonly encountered green anole found throughout much of Cuba."

Formation of descriptive or modifying words. - When a descriptor refers to a feature of an animal, the suffix -ed will be added. The modifying word will be treated as an adjective as opposed to a noun in apposition and hyphenation will be used in preference to combining two words (e.g., Cuban Flat-headed frog and Red-footed Tortoise). Exceptions to using a hyphen include the use of locations, geographic regions, or directions (e.g., Anguilla Bank Tree Anole or Lesser Antillean Iguana). The adjectival form of a name (e.g., Cuban Racer not Cuba Racer or Bahamian Racer not Bahamas Racer) should be used except when the use of an adjectival form would be awkward, excessively long, or imply an incorrect pronunciation. For example, Sierra Maestrae Bearded Anole not Sierra Maestraean Bearded Anole, Saint Croix Anole not Saint Crucian Anole, and Trinidad and Tobago Frog not Trinidanian and Tobagonian Frog. 
The names of genera and all higher categories should be plural.-Compound names, including those used in species names, may be spelled as a single word, unhyphenated, if the first component is a single syllable and the second component: (a) is among the words boa, frog, lizard, snake, toad, tortoise, turtle (e.g., Treeboa, Landfrog, Groundlizard, Blindsnake, or Watersnake); (b) refers to a body part (e.g., Curlytail, Whiptail); (c) describes an activity (e.g., Pondslider), or (d) is a misnomer (e.g., Coachwhip). Exceptions would be names ordinarily spelled as single unhyphenated words under the above rules that instead should be: (a) spelled as separate words with both capitalized when spelling as a single word would be awkward, (b) have three consecutive vowels or a triple-letter sequence, (c) be excessively long, or (d) imply an incorrect pronunciation (e.g., Tree Anole not Treeanole or House Gecko not Housegecko). Note that compound names not spelled as single words should have each word capitalized.

Names of species (and subspecies) should be singular.-The universal convention for common names of plants, animals, and fungi has been to treat each species in the singular form, and we are not aware of any exceptions. Nonetheless, de Queiroz (2011) argued that species names should be plural because species are multiple populations (or metapopulations) that extend through time. However, we do not consider this argument relevant because the name of a species, in this context, is the object of classification, not an entity comprised of individuals or populations. Deciding upon grammatical number for a common name is a linguistic decision not an evolutionary one.

Words shall be in English unless the name of a location or geographic region is derived from another language.-Note that words with accents in the language of origin should be spelled with accents only if necessary to indicate correct pronunciation in English.

In the following checklist, we have organized names alphabetically according to class (Amphibia, Reptilia), order (Anura, Crocodylia, Squamata, Testudines), family, and genus. We often employed widely used English names, but did not feel bound by tradition, and coined new names that were more informative or accurate (e.g., replacing patronyms with descriptive names and substituting more accurate geographic descriptors when appropriate). In order to avoid excessively long names, we sometimes used diminutives (e.g., Geckolet and Tegulet) instead of longer alternatives (e.g., Dwarf Gecko and Spectacled Tegu, respectively). We attempted to use names that would not overlap with English names of species from elsewhere in the Americas, but acknowledge that we might have overlooked some possible conflicts. Because standardized English names must change to accommodate new scientific names and combinations, we anticipate that this list will change in the future (as have such lists for other regions). However, we hope that, as attempts to develop standardized English names for all American amphibians and reptiles move forward, efforts will be made to complement existing lists, avoid parochial names applicable to only one area for wide-ranging species, and use names that will not preoccupy alternatives more suitable for species elsewhere in the Western Hemisphere.

We have listed currently recognized subspecies where appropriate, but only those known to occur on Caribbean islands. However, we have made no effort to provide standardized English names for subspecific taxa. We also inserted comments to identify extinct species (including named species known only from fossils) or to acknowledge differences of opinion for some taxonomic choices.

\section{Amphibia Latreille 1806-Salamanders, Frogs \& Toads, Caecilians}

\section{Anura Duméril 1806-Frogs \& Toads}

\section{Aromobatidae Grant, Frost, Caldwell, Gagliardo, Haddad, Kok, Means, Noonan, Schargel, \& Wheeler 2006-Cryptic Poison Frogs}

\section{Allobates Zimmermann \& Zimmermann 1988-White-lined Poison Frogs}

A. chalcopis (Kaiser, Coloma, \& Gray 1994)—Martinique Volcano Frog 


\section{Mannophryne La Marca 1992-Fingered Poison Frogs}

M. olmonae (Hardy 1983)-Tobago Poison Frog

M. trinitatis (Garman 1888)—Trinidad Poison Frog

\section{Bufonidae Gray 1825-Typical Toads}

\section{Incilius Cope 1863-Central American Toads}

I. valliceps (Wiegmann 1833)-Southern Gulf Coast Toad

\section{Peltophryne Fitzinger 1843-Caribbean Toads}

P. armata Landestoy T., Turner, Marion, \& Hedges 2018-Hispaniolan Armored Toad

P. cataulaciceps (Schwartz 1959)—Cuban Pineland Toad

P. dunni (Barbour 1926)—Central Cuban Long-nosed Toad

Comment. This taxon was described as a distinct species but has been recognized more recently as a subspecies of $P$. longinasus, although not based on any comprehensive analysis or evidence of hybridization. We recognize $P$. dunni as a distinct species because of its genetic, morphological, and geographical distinctiveness. Peltophryne dunni occurs $\sim 500 \mathrm{~km}$ east of $P$. longinasus, and $\sim 500 \mathrm{~km}$ west of $P$. ramsdeni. Alonso et al. (2012) showed that the sequence of $P$. dunni (AY028493) reported by Pramuk et al. (2001) is phylogenetically more distant than expected, although they had limited sampling and no material from P. ramsdeni. Suprisingly, they treated sequence AY028493 as belonging to $P$. longinasus, rendering that species polyphyletic. They explained this unusual taxonomic decision by suggesting that the sequence must be from an undescribed species distinct from $P$. dunni, because of its slightly more distant position in the tree. Although the sample could represent an undescribed species, the specimen was identified initially as $P$. dunni and was collected within the narrow range of that species. Given that no other sequences of $P$. dunni exist, and without further information, we believe that it is more appropriate to assume that sequence AY028493 represents $P$. dunni, a valid species. The taxon P. longinasus cajalbanensis was described as a subspecies of the geographically proximal $P$. longinasus. Alonso et al. (2012) found the two taxa were close genetically as well, and therefore we concur in treating $P$. I. cajalbanensis as a subspecies of $P$. longinasus.

P. empusa Cope 1862-Cuban Small-eared Toad

P. florentinoi (Moreno \& Rivalta 2007)—Zapata Toad

P. fluviatica (Schwartz 1972)—Hispaniolan Crestless Toad

P. fustiger (Schwartz 1960)—Western Cuba Giant Toad

P. guentheri (Cochran 1941)—Southern Hispaniola Crested Toad

P. gundlachi (Ruibal 1959)—Cuban High-crested Toad

P. lemur Cope 1868-Puerto Rican Toad

P. longinasus (Stejneger 1905)—Western Cuba Long-nosed Toad

Subspecies. Peltophryne longinasus longinasus (Stejneger 1905); P. I. cajalbanensis (Valdés de la Osa and Ruiz-Garcia 1980).

P. peltocephala (Tschudi 1838)-Eastern Cuba Giant Toad

P. ramsdeni (Barbour 1914)—Eastern Cuba Long-nosed Toad

Comment. See comment for $P$. dunni. Peltophyrne ramsdeni was described as a distinct species. Although often considered a subspecies of $P$. longinasus, we recognize this taxon as a species because of its morphological and geographical distinctiveness when compared to other taxa associated with $P$. longinasus. For example, $P$. dunni is phylogenetically removed from any subspecific association with $P$. longinasus (Alonso et al. (2012) and $P$. ramsdeni is even farther removed, at least geographically, being $>1000 \mathrm{~km}$ east of the range of $P$. longinasus. No genetic data exist for $P$. ramsdeni.

P. taladai (Schwartz 1960)—Cuban Spotted Toad 


\section{Rhinella Fitzinger 1826-South American Toads}

R. beebei (Gallardo 1965)—North Coastal Granular Toad

R. marina (Linnaeus 1758)-South American Cane Toad

Comment. As traditionally defined, Rhinella marina is a complex of several species (e.g., Solis et al., 2009). Vallinoto et al. (2010) provided molecular evidence that the Amazonian population to which the name R. marina was originally assigned is closely related to other members of the complex (the $R$. marina group of Maciel et al., 2010) but distant from clades in Central America and western Ecuador. Acevedo et al. (2016) referred the latter, and populations north through Mexico and into the Lower Rio Grande Valley of Texas, to the Mesoamerican Cane Toad (R. horribilis).

\section{Centrolenidae Taylor 1951-Glassfrogs}

Hyalinobatrachium Ruiz-Carranza \& Lynch 1991-Neotropical Glassfrogs

H. orientale (Rivero 1968)—Eastern Glass Frog

\section{Eleutherodactylidae Lutz 1954-Greater Caribbean Landfrogs}

\section{Eleutherodactylus Duméril \& Bibron 1841-Caribbean \& Mexican Landfrogs}

E. abbotti Cochran 1923-Common Chirping Frog

E. acmonis Schwartz 1960-Cuban Red-rumped Frog

E. adelus Díaz, Cádiz, \& Hedges 2003-Cuban Dwarf Grassfrog

E. albipes Barbour \& Shreve 1937-Turquino White-footed Frog

E. alcoae Schwartz 1971—Barahona Rockfrog

E. alticola Lynn 1937-Jamaican Peak Frog

E. amadeus Hedges, Thomas, \& Franz 1987-Macaya Bushfrog

E. amplinympha Kaiser, Green, \& Schmid 1994 (1995)—Dominica Frog

E. andrewsi Lynn 1937-Jamaican Rump-spotted Frog

E. antillensis (Reinhardt \& Lütken 1862)—Puerto Rican Red-eyed Frog

E. aporostegus Schwartz 1965-Tiburon Burrowing Frog

E. apostates Schwartz 1973-La Hotte Big-legged Frog

E. armstrongi Noble \& Hassler 1933—Baoruco Hammer Frog

E. atkinsi Dunn 1925-Cuban Groin-spotted Frog

E. audanti Cochran 1934-South Island Telegraphic Frog

E. auriculatoides Noble 1923-Northern Hammer Frog

E. auriculatus (Cope 1862)—Cuban Telegraphic Frog

E. bakeri Cochran 1935-La Hotte Bushfrog

E. barlagnei Lynch 1965-Guadeloupe Streamfrog

E. bartonsmithi Schwartz 1960—Boca De Yumuri Frog

E. blairhedgesi Estrada, Díaz, \& Rodríguez 1997-Canasi Frog

E. bothroboans Schwartz 1965-Cordillera Central Frog

E. bresslerae Schwartz 1960-Maisi Frog

E. brevirostris Shreve 1936-Short-nosed Green Frog

E. brittoni Schmidt 1920—Puerto Rican Grassfrog

E. caribe Hedges \& Thomas 1992-Haitian Marshfrog

E. casparii Dunn 1926-Trinidad Flat-headed Frog

E. cavernicola Lynn 1954-Portland Ridge Frog

E. chlorophenax Schwartz 1976—La Hotte Giant Frog 
E. cochranae Grant 1932-Puerto Rican Whistling Frog

E. cooki Grant 1931—Puerto Rican Cave Frog

E. coqui Thomas 1966-Puerto Rican Coqui

E. corona Hedges \& Thomas 1992-Hispaniolan Crowned Frog

E. counouspeus Schwartz 1964-Yellow Cave Frog

E. cubanus Barbour 1942-Turquino Red-armed Frog

E. cundalli Dunn 1926-Jamaican Rockfrog

E. cuneatus (Cope 1862)—Oriente Streamside Frog

E. darlingtoni Cochran 1935-La Selle Long-legged Frog

E. dimidiatus (Cope 1862)—Cuban Long-legged Frog

Subspecies. Eleutherodactylus dimidiatus dimidiatus (Cope 1862); E. d. amelasma Schwartz 1958.

E. diplasius Schwartz 1973-Patternless Whistling Frog

E. dolomedes Hedges \& Thomas 1992-Haitian Ventriloquial Frog

E. eileenae Dunn 1926-Cuban Two-note Frog

E. emiliae Dunn 1926-Trinidad Groin-spotted Frog

E. eneidae Rivero 1959-Puerto Rican Elegant Frog

E. erythroproctus Schwartz 1960—Camarones Red-legged Frog

E. etheridgei Schwartz 1958-Oriente Coastal Frog

E. eunaster Schwartz 1973-La Hotte Whistling Frog

E. feichtingeri Díaz, Hedges, \& Schmid 2012-Eastern Cuba Grassfrog

E. flavescens Noble 1923-Yellow Split-toed Frog

E. fowleri Schwartz 1973-Khaki Bromeliad Frog

E. furcyensis Shreve \& Williams 1963—La Selle Red-legged Frog

E. fuscus Lynn \& Dent 1942-Jamaican Ear-spotted Frog

E. glamyrus Estrada \& Hedges 1997-Turquino Fern Frog

E. glandulifer Cochran 1935-La Hotte Glanded Frog

E. glanduliferoides Shreve 1936-La Selle Grassfrog

E. glaphycompus Schwartz 1973-Ball Bearing Frog

E. glaucoreius Schwartz \& Fowler 1973-Blue Mountain Rockfrog

E. goini Schwartz 1960-Guaniguanico Yellow-mottled Frog

E. gossei Dunn 1926-Jamaican Forest Frog

Subspecies. Eleutherodactylus gossei gossei Dunn 1926; E. g. oligaulax Schwartz \& Fowler 1973.

E. grabhami Dunn 1926-Jamaican Pallid Frog

E. grahami Schwartz 1979-Yellow Chevronate Frog

E. greyi Dunn 1926-Cuban Gray Frog

E. griphus Crombie 1986-Cockpit Frog

E. gryllus Schmidt 1920—Puerto Rican Green Frog

E. guanahacabibes Estrada \& Novo Rodríguez 1985-Guanahacabibes Frog

E. guantanamera Hedges, Estrada, \& Thomas 1992-Guantanamera Frog

E. gundlachi Schmidt 1920-Turquino Spiny Frog

E. haitianus Barbour 1942-Montane Cricket Frog

E. hedricki Rivero 1963-Puerto Rican Treehole Frog

E. heminota Shreve \& Williams 1963-Half-striped Bromeliad Frog

E. hypostenor Schwartz 1965-Baoruco Burrowing Frog

E. iberia Estrada \& Hedges 1996-Monte Iberia Dwarf Frog

E. inoptatus Barbour 1914-Hispaniolan Giant Frog 
E. intermedius Barbour \& Shreve 1937-Oriente Dark-bellied Frog

E. ionthus Schwartz 1960-Oriente Treefrog

E. jamaicensis Barbour 1910—Jamaican Bromeliad Frog

E. jasperi Drewry \& Jones 1976—Puerto Rican Golden Frog

E. jaumei Estrada \& Alonso 1997-Orange Long-nosed Frog

E. johnstonei Barbour 1914-Lesser Antillean Frog

E. juanariveroi Rios-López \& Thomas 2007—Puerto Rican Wetland Frog

E. jugans Cochran 1937-La Selle Dusky Frog

E. junori Dunn 1926-Rock Pocket Frog

E. karlschmidti Grant 1931—Puerto Rican Streamfrog

E. klinikowskii Schwartz 1959-Barred Rockfrog

E. lamprotes Schwartz 1973-Hispaniolan Orange-legged Frog

E. leberi Schwartz 1965-Oriente Yellow-bellied Frog

E. lentus (Cope 1862)—Virgin Islands Yellow Frog

E. leoncei Shreve \& Williams 1963-Southern Pastel Frog

E. ligiae Incháustegui, Díaz, \& Marte 2015-Baoruco Rockfrog

E. limbatus (Cope 1862)—Yellow-striped Dwarf Frog

E. limbensis Lynn, 1958-Haitian Streamside Frog

E. locustus Schmidt 1920—Puerto Rican Small-eared Frog

E. lucioi Schwartz 1980—Pallid Rockfrog

E. luteolus (Gosse 1851)—Jamaican Masked Frog

E. maestrensis Díaz, Cádiz, \& Navarro 2005-Sierra Maestra Long-legged Frog

E. mariposa Hedges, Estrada, \& Thomas 1992—Cuban Butterfly-marked Frog

E. martinicensis (Tschudi 1838)—Martinique Frog

E. melacara Hedges, Estrada, \& Thomas 1992-Dark-faced Bromeliad Frog

E. melatrigonum Schwartz 1966-Cordillera Central Telegraphic Frog

E. michaelschmidi Díaz, Cádiz, \& Hedges 2007-Sierra Maestra Blotched Frog

E. minutus Noble 1923-Hispaniolan Wheeping Frog

E. monensis (Meerwarth 1901)—Mona Frog

E. montanus Schmidt 1919-Hispaniolan Montane Frog

E. neiba Incháustegui, Díaz, \& Marte 2015-Neiba Leaf-litter Frog

E. nortoni Schwartz 1976-Spiny Giant Frog

E. notidodes Schwartz 1966-Neiba Telegraphic Frog

E. nubicola Dunn 1926-Jamaican Red-eyed Frog

E. olibrus Schwartz 1958-Pinar Del Rio Bromeliad Frog

E. orcutti Dunn 1928-Jamaican Streamfrog

E. orientalis (Barbour \& Shreve 1937)—Baracoa Dwarf Frog

E. oxyrhyncus (Duméril \& Bibron 1841)—Hispaniolan Sharp-nosed Frog

E. pantoni Dunn 1926-Jamaican Yellow-bellied Frog

Subspecies. Eleutherodactylus pantoni pantoni Dunn 1926; E. p. amiantus Schwartz \& Fowler 1973.

E. parabates Schwartz 1964-Neiba Whistling Frog

E. paralius Schwartz 1976-Coastal Red-rumped Frog

E. parapelates Hedges \& Thomas 1987-Macaya Burrowing Frog

E. patriciae Schwartz 1964 (1965)—Hispaniolan Cordillera Frog

E. paulsoni Schwartz 1964-Hispaniolan Pink-rumped Frog

E. pentasyringos Schwartz \& Fowler 1973-John Crow Yellow-bellied Frog 
E. pezopetrus Schwartz 1960-Eastern Cuba Rockfrog

E. pictissimus Cochran 1935-Hispaniolan Yellow-mottled Frog

Subspecies. Eleutherodactylus pictissimus pictissimus Cochran 1935; E. p. apantheatus Schwartz 1965; E. p. eremus Schwartz 1965.

E. pinarensis Dunn 1926—Cuban Pineland Frog

E. pinchoni Schwartz 1967-Guadeloupe Forest Frog

E. pituinus Schwartz 1964-Hispaniolan Melodius Frog

E. planirostris (Cope 1862)—Cuban Flat-headed Frog

E. poolei Cochran 1938-Citadel Frog

E. portoricensis Schmidt 1927-Puerto Rican White-eyed Frog

E. principalis Estrada \& Hedges 1997-Oriente Greenish-yellow Frog

E. probolaeus Schwartz 1965-Boca De Yuma Frog

E. rhodesi Schwartz 1980-Port-De-Paix Frog

E. richmondi Stejneger 1904-Puerto Rican Bronze Frog

E. ricordii (Duméril \& Bibron 1841)—Oriente Yellow-mottled Frog

E. riparius Estrada \& Hedges 1998-Cuban Streamside Frog

E. rivularis Díaz, Estrada, \& Hedges 2001—Short-legged Streamside Frog

E. rogersi Goin 1955-Bahamian Flat-headed Frog

E. ronaldi Schwartz 1960-Cuban Khaki Frog

E. rucillensis Cochran 1939—Rucilla Streamside Frog

E. rufifemoralis Noble \& Hassler 1933-Baoruco Red-legged Frog

E. ruthae Noble 1923-Eastern Burrowing Frog

E. schmidti Noble 1923-Hispaniolan Streamside Frog

E. schwartzi Thomas 1966-Virgin Islands Khaki Frog

E. sciagraphus Schwartz 1973-La Hotte Stripe-legged Frog

E. semipalmatus Shreve 1936-Tiburon Streamfrog

E. simulans Díaz \& Fong 2001—Oriente Mottled Frog

E. sisyphodemus Crombie 1977—Leaf Mimic Frog

E. sommeri Schwartz 1977-Massif Du Nord Whistling Frog

E. staurometopon Schwartz 1960-Isla De La Juventud Frog

E. symingtoni Schwartz 1957-Western Spiny Frog

E. tetajulia Estrada \& Hedges 1996-Cuban Stripeless Leaf-litter Frog

E. thomasi Schwartz 1959-Cuban Cave Frog

Subspecies. Eleutherodactylus thomasi thomasi Schwartz 1959; E. t. trinidadensis Schwartz 1959; E. t. zayasi Schwartz 1960.

E. thorectes Hedges 1988-Macaya Breast-spotted Frog

E. toa Estrada \& Hedges 1991—Oriente Pallid Frog

E. tonyi Estrada \& Hedges 1997-Cabo Cruz Frog

E. turquinensis Barbour \& Shreve 1937-Turquino Streamfrog

E. tychathrous Schwartz 1965-Vallejuelo Burrowing Frog

E. unicolor Stejneger 1904-Puerto Rican Elfin Frog

E. varians (Gundlach \& Peters in Peters 1864)—Cuban Bromeliad Frog

E. varleyi Dunn 1925-Western Cuba Grassfrog

E. ventrilineatus (Shreve 1936)—Macaya Dusky Frog

E. warreni Schwartz 1976-Tortue Frog

E. weinlandi Barbour 1914-Hispaniolan Red-rumped Frog 
Subspecies. Eleutherodactylus weinlandi weinlandi Barbour 1914; E. w. chersonesodes Schwartz 1965.

E. wetmorei Cochran 1932-Tiburon Whistling Frog

Subspecies. Eleutherodactylus wetmorei wetmorei Cochran 1932; E. w. ceraemerus Schwartz 1968.

E. wightmanae Schmidt 1920—Puerto Rican Melodius Frog

E. zeus Schwartz 1958-Cuban Giant Frog

E. zugi Schwartz 1958-Rosario Red-legged Frog

\section{Hemiphractidae Peters 1862-Marsupial Frogs}

Flectonotus Miranda-Ribeiro 1926-Venezuelan Marsupial Frogs

F. fitzgeraldi (Parker 1933)—Dwarf Marsupial Frog

\section{Hylidae Rafinesque 1815-Typical Treefrogs}

\section{Agalychnis Cope 1864-Middle American Multicolored Treefrogs}

A. callidryas (Cope 1862)—Red-eyed Multicolored Treefrog

\section{Boana Gray 1825-Gladiator Treefrogs}

B. boans (Linnaeus 1758)-Rusty Treefrog

B. geographica (Spix 1824)—Map Treefrog

B. heilprini (Noble 1923)—Hispaniolan Green Treefrog

B. punctata (Schneider 1799)—Dotted Treefrog

B. xerophylla (Duméril \& Bibron 1841)—Emerald-eyed Treefrog

\section{Dendropsophus Fitzinger 1843-Dwarf Treefrogs}

D. goughi (Boulenger 1911)—Guianan Dwarf Treefrog

Comment. We follow Gehara et al. (2014) in recognizing D. goughi as a valid species.

D. microcephalus (Cope 1886)—Small-headed Dwarf Treefrog

D. minusculus (Rivero 1971)—Pallid Dwarf Treefrog

D. phlebodes (Stejneger 1906)—San Carlos Dwarf Treefrog

\section{Dryophytes Fitzinger 1843-Ameroasian Treefrogs}

D. cinereus (Schneider 1799)—North American Green Treefrog

D. squirellus (Bosc in Daudin 1800)—Squirrel Treefrog

\section{Osteopilus Fitzinger 1843-North Caribbean Treefrogs}

O. crucialis (Harlan 1826)—Jamaican Snoring Treefrog

O. dominicensis (Tschudi 1838)—Hispaniolan Laughing Treefrog

O. marianae (Dunn 1926)_Jamaican Yellow Treefrog

O. ocellatus (Linnaeus 1758)_Jamaican Laughing Treefrog

O. pulchrilineatus (Cope 1869)—Hispaniolan Yellow Treefrog

O. septentrionalis (Duméril \& Bibron 1841)—Cuban Treefrog

O. vastus (Cope 1871)—Hispaniolan Giant Treefrog

O. wilderi (Dunn 1925)—Jamaican Green Treefrog

\section{Phyllomedusa Wagler 1830-South American Multicolored Treefrogs}

P. trinitatis Mertens 1926-Trinidad Leaf-nesting Frog

Phytotriades Jowers, Downieb, \& Cohen 2009-Trinidad Golden Treefrogs

P. auratus (Boulenger 1917)—Trinidad Golden Treefrog 


\section{Pseudis Wagler 1830-Robust South American Swimming Frogs}

P. paradoxa (Linnaeus 1758)—Paradox Frog

\section{Scarthyla Duellman \& de Sá 1988-South American Aquatic Treefrogs}

S. vigilans (Solano 1971)—Maracaibo Basin Treefrog

\section{Scinax Wagler 1830-American Snouted Treefrogs}

S. ruber (Laurenti 1768)—Common Snouted Treefrog

Comment. This taxon probably is a complex of species (Solis et al. 2010).

S. staufferi (Cope 1865)—Middle American Snouted Treefrog

S. $x$-signatus (Spix 1824)—Venezuelan Snouted Treefrog

\section{Smilisca Cope 1865-Middle American Treefrogs}

S. baudinii (Duméril \& Bibron 1841)—Common Mexican Treefrog

\section{Sphaenorhynchus Tschudi 1838-Short-snouted Green Treefrogs}

S. lacteus (Daudin 1800)—Ghost Frog

Tlalocohyla Faivovich, Haddad, Garcia, Frost, Campbell, \& Wheeler 2005-Middle American Yellow-bellied Treefrogs

T. loquax (Gaige \& Stuart 1934)—Mahogany Treefrog

Trachycephalus Tschudi 1838-Casque-headed Canopy Treefrogs

T. typhonius (Linnaeus 1758)—Pepper Treefrog

\section{Leptodactylidae Werner 1896 (1838)—Neotropical Thin-toed Frogs}

\section{Adenomera Steindachner 1867-Robust Thin-toed Frogs}

A. hylaedactyla (Cope 1868)—Dark-spotted Thin-toed Frog

Comment. This taxon might represent a species complex, within which the species in our region has yet to be named and might best be referenced as "Adenomera sp." (J.C. Murphy, unpubl.).

\section{Engystomops Jiménez de la Espada 1872-Neotropical Puddlefrogs}

E. pustulosus (Cope 1864)—Tungara Frog

\section{Leptodactylus Fitzinger 1826-Neotropical White-lipped Frogs}

L. albilabris (Günther 1859)—Antillean White-lipped Frog

L. fallax Müller 1926-Mountain Chicken

L. fragilis (Brocchi 1877)—Middle American White-lipped Frog

L. fuscus (Schneider 1799)—South American White-lipped Grassfrog

L. insularum (Barbour 1906)—Caribbean Ditchfrog

L. latrans (Steffen 1815)-South American Spotted Grassfrog

L. melanonotus (Hallowell 1861)—Reddish-brown White-lipped Frog

L. nesiotus Heyer 1994-Trinidad Ditchfrog

L. validus Garman 1887-Windward Islands Ditchfrog

Pleurodema Tschudi 1838-Four-eyed Frogs

P. brachyops (Cope 1869)—Stout Four-eyed Frog

Microhylidae Günther 1858 (1843)—Narrow-mouthed Frogs

Elachistocleis Parker 1927-Oval Frogs 
E. ovalis (Schneider 1799)—Northern Oval Frog

E. surinamensis (Daudin 1802)—Northeastern Oval Frog

Gastrophryne Fitzinger 1843-North American Narrow-mouthed Frogs

G. carolinensis (Holbrook 1836)—Eastern Narrow-mouthed Frog

\section{Pipidae Gray 1825-Tongueless Frogs}

Pipa Laurenti 1768-Neotropical Watertoads

P. pipa (Linnaeus 1758)—Star-fingered Frog

\section{Ranidae Batsch 1796-Waterfrogs}

\section{Lithobates Fitzinger 1843-North American Waterfrogs}

L. brownorum (Sanders 1973)—Central American Waterfrog

L. catesbeianus (Shaw 1802)-American Bullfrog

L. grylio (Stejneger 1901)—American Pigfrog

L. miadis (Barbour \& Loveridge 1929)—Little Corn Island Frog

L. palmipes (Spix 1824)—Amazon Waterfrog

Comment. This taxon is a complex of species (La Marca et al. 2010).

L. sphenocephalus (Cope 1886)—Southern Leopard Frog

L. vaillanti (Brocchi 1877)—Common Marshfrog

Comment. This taxon is a complex of species (Santos-Barrera et al. 2008).

\section{Strabomantidae Hedges, Duellman, \& Heinicke 2008-South American Landfrogs}

\section{Pristimantis Jiménez de la Espada 1870-South American Big-toed Landfrogs}

P. charlottevillensis (Kaiser, Dwyer, Feichtinger, \& Schmidt 1995)—Charlotteville Leaf-litter Frog

P. euphronides (Schwartz 1967)—Grenada Frog

P. shrevei (Schwartz 1967)—Saint Vincent Frog

P. turpinorum (Hardy 2001)—Tobago Long-legged Palmfrog

P. urichi (Boettger 1894)—Trinidad \& Tobago Frog

\section{Caudata Scopoli 1777-Salamanders}

\section{Plethodontidae Gray 1850_Lungless Salamanders}

\section{Palaeoplethodon Poinar \& Wake 2015-Hispaniolan Amber Salamanders}

P. hispaniolae Poinar \& Wake 2015-Hispaniolan Amber Salamander

Comment. This genus and species are known from only a single specimen in Dominican amber (Poinar \& Wake 2015).

\section{Reptilia Laurenti 1768-Amphisbaenians, Lizards, Snakes, Turtles, Alligators \& Crocodiles}

\section{Squamata Oppel 1811-Amphisbaenians, Lizards, Snakes}

Comment. Amphisbaenians, lizards, and snakes once were considered formal taxonomic entities: Suborder Amphisbaenia for wormlizards, Lacertilia or Sauria for lizards, and Serpentes or Ophidia for snakes. However, phylogenetic studies clearly show that snakes and wormlizards are specialized legless lizards. We retain the distinction 
between amphisbaenians, lizards, and snakes solely for convenience. In the Caribbean region (but not necessarily everywhere), what we call "amphisbaenians" always have rings of scales that circle the body, "lizards" always have at least one of the following: movable eyelids, limbs, or external ears, whereas "snakes" lack limbs, movable eyelids, and external ears, and scales around the body never form distinct rings.

\section{Squamata Oppel 1811 (part)-Amphisbaenians}

Family Amphisbaenidae Gray 1865-Wormlizards

\section{Amphisbaena Linnaeus 1758-Typical Wormlizards}

A. alba Linnaeus 1758-Red Wormlizard

A. bakeri Stejneger 1904-Puerto Rican Many-ringed Wormlizard

A. barbouri Gans \& Alexander 1962—Cuban Many-ringed Wormlizard

A. caeca Cuvier 1829-Common Puerto Rican Wormlizard

A. carlgansi Thomas \& Hedges 1998-Cuban Pink Wormlizard

A. caudalis Cochran 1928-Cayemite Long-tailed Wormlizard

A. cayemite Thomas \& Hedges 2006-Cayemite Short-tailed Wormlizard

A. cubana Gundlach \& Peters in Peters 1879 ("1878")—Cuban Brown Wormlizard

A. fenestrata (Cope 1861) - Virgin Islands Wormlizard

A. fuliginosa Linnaeus 1758-Speckled Wormlizard

Subspecies. Amphisbaena fuliginosa fuliginosa Linnaeus 1758.

A. gonavensis Gans \& Alexander 1962-Gonave Wormlizard

A. hyporissor Thomas 1965-Barahona Wormlizard

A. innocens Weinland 1862-Tiburon Wormlizard

A. leali Thomas \& Hedges 2007 ("2006")—Pestel Wormlizard

A. manni Barbour 1914-Hispaniolan Dwarf Wormlizard

A. schmidti Gans 1964-Puerto Rican Dusky Wormlizard

A. xera Thomas 1966—Puerto Rican Xeric Wormlizard

\section{Family Cadeidae Vidal \& Hedges 2008—Cuban Keel-headed Wormlizards}

\section{Cadea Gray 1844-Cuban Wormlizards}

C. blanoides (Stejneger 1916)—Cuban Spotted Wormlizard

C. palirostrata Dickerson 1916-Cuban Sharp-nosed Wormlizard

\section{Squamata Oppel 1811 (part)—Lizards}

\section{Family Anguidae Gray 1825-Alligator Lizards, Galliwasps, Glass Lizards}

\section{Celestus Gray 1839-Sheathless Galliwasps}

C. agasepsoides (Thomas 1971)-Serpentine Four-toed Galliwasp

C. anelpistus (Schwartz, Graham, \& Duval 1979)—Altagracia Giant Galliwasp

C. badius Cope 1868-Navassa Galliwasp

C. barbouri Grant 1940-Limestone Forest Galliwasp

C. costatus (Cope 1862 ["1861"])—Hispaniolan Smooth Galliwasp

Subspecies. Celestus costatus costatus (Cope 1862 ["1861"]); C. c. chalcorhabdus (Schwartz 1964); C. c. emys (Schwartz 1971); C. c. leionotus (Schwartz 1964); C. c. melanchrous (Schwartz 1964); C. c. neiba (Schwartz 1964); C. c. nesobous (Schwartz 1964); C. c. oreistes (Schwartz 1964); C. c. psychonothes (Schwartz 1964); C. c. saonae 
(Schwartz 1971).

C. crusculus (Garman 1888)_Jamaican Galliwasp

Subspecies. Celestus crusculus crusculus (Garman 1888); C. c. cundalli Grant 1940.

C. curtissi Grant 1951—Hispaniolan Khaki Galliwasp

Subspecies. Celestus curtissi curtissi Grant 1951; C. c. aporus (Schwartz 1964); C. c. diastatus (Schwartz 1964); C.

c. hylonomus (Schwartz 1964).

C. darlingtoni Cochran 1939-Hispaniolan Striped Galliwasp

C. duquesneyi Grant 1940—Blue-tailed Galliwasp

C. fowleri (Schwartz 1971)—Bromeliad Galliwasp

C. haetianus (Cochran 1927)—Hispaniolan Earless Galliwasp

Subspecies. Celestus haetianus haetianus (Cochran 1927); C. h. mylicus (Schwartz 1965); C. h. surdus (Schwartz 1965).

C. hewardii Gray 1845-Red-spotted Galliwasp

C. macrotus Thomas \& Hedges 1989—La Selle Galliwasp

C. maculatus (Garman 1887)—Cayman Galliwasp

C. marcanoi (Schwartz \& Incháustegui 1976)—Pico Duarte Galliwasp

C. microblepharis (Underwood 1959)—Small-eyed Galliwasp

C. molesworthi Grant 1940_Portland Coast Galliwasp

C. occiduus (Shaw 1802) - Jamaican Giant Galliwasp

Comment. This taxon presumably is extinct (Wilson et al. 2017).

C. sepsoides (Gray 1852) —Hispaniolan Four-toed Galliwasp

C. stenurus (Cope 1863 ["1862"])—Hispaniolan Keeled Galliwasp

Subspecies. Celestus stenurus stenurus (Cope 1863 ["1862"]); C. s. alloeides (Schwartz 1964); C. s. rugosus Cope 1879; C. s. weinlandi Cope 1868.

C. warreni (Schwartz 1970)—Hispaniolan Giant Galliwasp

\section{Diploglossus Wiegmann 1834-Sheathed Galliwasps}

D. delasagra (Cocteau 1838-1843)—Cuban Pale-necked Galliwasp

D. garridoi Thomas \& Hedges 1998—Cuban Small-eared Galliwasp

D. montisserrati Underwood 1964-Montserrat Galliwasp

Comment. Bochaton et al. (2016) described fossils from Guadeloupe (both Grande-Terre and Basse-Terre) that are very similar to $D$. montisserrati but exhibit some minor morphological differences; the authors stated that the differences were insufficient to warrant the description of a new species and referred the fossils to Diploglossus sp.

D. nigropunctatus (Barbour \& Shreve 1937)—Cuban Spotted Galliwasp

D. pleii Duméril \& Bibron 1839—Puerto Rican Galliwasp

Family Corytophanidae Fitzinger 1843-Basilisks, Casque-headed Lizards, Helmeted Lizards

Basiliscus Laurenti 1768-Basilisks

B. vittatus Wiegmann 1828-Brown Basilisk

Corytophanes H. Boie in Schlegel 1826-Helmeted Lizards

C. cristatus (Merrem 1820)—Smooth Helmeted Lizard

Family Dactyloidae Fitzinger 1843-Typical Anoles

Anolis Daudin 1802-Typical Anoles 
Comment. Several proposals to recognize five or even eight anoline genera (Guyer \& Savage 1986; Savage \& Guyer 1989; Nicholson et al. 2012) have been most frequently applied by authors working with mainland species, but have generally not been accepted (e.g., Williams 1989; Poe 2013). Other authors (e.g., Castañeda \& de Queiroz 2011; de Queiroz et al. 2017; Kraus \& Krysko 2017; Poe et al. 2017) have used the name Anolis for the entire clade, but applied the other names to subclades sometimes in ways that differed from the earlier generic assignments. Recently, Nicholson et al. (2018) reignited this debate and many taxonomists who work with reptiles appear to be leaning toward a split of Anolis into eight genera. In the following list, we do not advocate either taxonomy but, for convenience here, retain the single genus Anolis in a full listing of species (alphabetical) but also include the alternative genus and species names in parentheses and correct some spelling errors in Nicholson et al. $(2012,2018)$. Note that some specific names differ in spelling because some of the new genera differ in gender from Anolis.

A. acutus (Ctenonotus actus) Hallowell 1856-Saint Croix Anole

A. aeneus (Dactyloa aenea) Gray 1840-Grenada Bush Anole

A. agueroi (Xiphosurus agueroi) (Díaz, Navarro, \& Garrido 1998)—Cabo Cruz Bearded Anole

A. ahli (Norops ahli) Barbour 1925-Escambray Blue-eyed Anole

A. alayoni Estrada \& Hedges 1995-Guantanamo Twig Anole

A. alfaroi Garrido \& Hedges 1992-Small-fanned Bush Anole

A. aliniger (Deiroptyx aliniger) Mertens 1939-Axillary Spotted Anole

A. allisoni Barbour 1928-Cuban Blue Anole

A. allogus (Norops allogus) Barbour \& Ramsden 1919-Spanish Flag Anole

A. altavelensis (Ctenonotus altavelensis) Noble \& Hassler 1933-Alto Velo Gracile Anole

A. altitudinalis Garrido 1985-Turquino Green-mottled Anole

A. alumina (Chamaelinorops alumina) Hertz 1976-Barahona Grass Anole

A. alutaceus Cope 1861-Blue-eyed Twig Anole

A. anfiloquioi Garrido 1980—Brown-eyed Bush Anole

A. angusticeps Hallowell 1856-Cuban Twig Anole

A. apletolepis (Deiropteryx apletolepis) Köhler \& Hedges 2016—La Selle Twig Anole

A. argenteolus Cope 1861—Cuban Trunk Anole

A. argillaceus Cope 1862-Cuban Dark Bark Anole

A. armouri (Audantia armouri) (Cochran 1934)—Black-throated Stout Anole

A. bahorucoensis (Deiroptyx bahorucoensis) Noble \& Hassler 1933-Baoruco Long-snouted Anole

Subspecies. Anolis bahorucoensis bahorucoensis (Deiroptyx bahorucoensis bahorucoensis) Noble \& Hassler 1933; A. b. southerlandi (D. b. southerlandi) Schwartz 1978.

A. baleatus (Xiphosurus baleatus) (Cope 1864)—Dominican Giant Anole

Subspecies. Anolis baleatus baleatus (Xiphosurus baleatus baleatus) (Cope 1864); A. b. altager (X. b. altager) Schwartz 1975; A. b. caeruleolatus (X. b. caeruleolatus) Schwartz 1974; A. b. fraudator (X. b. fraudator) Schwartz 1974; A. b. lineatacervix (X. b. lineatacervix) Schwartz 1978; A. b. litorisilva (X. b. litorisilva) Schwartz 1974; A. b. multistruppus (X. b. multistruppus) Schwartz 1974; A. b. samanae (X. b. samanae) Schwartz 1974; A. b. scelestus (X. b. scelestus) Schwartz 1974; A. b. sublimis (X. b. sublimis) Schwartz 1974.

A. baracoae (Deiroptyx baracoae) Schwartz 1964-Baracoa Giant Anole

A. barahonae (Xiphosurus barahonae) Williams 1962-Baoruco Giant Anole

Subspecies. Anolis barahonae barahonae (Xiphosurus barahonae barahonae) Williams 1962; A. b. albocellatus (X. b. albocellatus) Schwartz 1974; A. b. ininquinatus (X. b. ininquinatus) Cullom \& Schwartz 1980; A. b. mulitus (X. b. mulitus) Cullom \& Schwartz 1980.

A. barbatus (Xiphosurus barbatus) (Garrido 1982)—Western Bearded Anole

A. barbouri (Chamaelinorops barbouri) (Schmidt 1919)—Hispaniolan Hopping Anole 
A. bartschi (Deiroptyx bartschi) (Cochran 1928)—Pinar Del Rio Cliff Anole

A. bicaorum (Norops bicaorum) (Köhler 1996)—Bay Islands Anole

A. bimaculatus (Ctenonotus bimaculatus) (Sparrman 1784)—Statia Bank Tree Anole

A. birama (Norops birama) Garrido 1990—Cuban Big-eared Anole

A. blanquillanus (Dactyloa blanquillana) Hummelinck 1940—La Blanquilla Anole

A. bonairensis (Dactyloa bonairensis) Ruthven 1923-Bonaire Anole

A. bremeri (Norops bremeri) Barbour 1914-Cuban Variegated Anole

Subspecies. Anolis bremeri bremeri (Norops bremeri bremeri) Barbour 1914; A. b. insulaepinorum (N. b. insulaepinorum) Schwartz 1978.

A. breslini (Audantia breslini) Schwartz 1980-Northwest Haitian Stout Anole

A. brevirostris (Ctenonotus brevirostris) Bocourt 1870-Desert Gracile Anole

Subspecies. Anolis brevirostris brevirostris (Ctenonotus brevirostris brevirostris) Bocourt 1870; A. b. deserticola (C. b. deserticola) Arnold 1980; A. b. wetmorei (C. b. wetmorei) Cochran 1931.

A. brunneus Cope 1895 ("1894")—Crooked-Acklins Green Anole

A. carolinensis Voight in Cuvier \& Voight 1832-North American Green Anole

Subspecies. Anolis carolinensis carolinensis Voight in Cuvier \& Voight 1832; A. c. seminolus Vance 1991.

Comment. At least two dispersal events led to the expansion of this species into North America (Tollis \& Boissinot 2014), and Tollis et al. (2012) found four distinct lineages, none of which corresponded to A. c. seminolus. Consequently, with evidence of considerable genetic and morphological variation throughout the distribution of this wide-ranging species, recognition of A. c. seminolus might not be justified.

A. caudalis (Ctenonotus caudalis) Cochran 1932-Gonave Gracile Anole

A. centralis Peters 1970-Central Pallid Anole

A. chamaeleonides (Xiphosurus chamaeleonides) Duméril \& Bibron 1837-Short-bearded Anole

A. chlorocyanus (Deiroptyx chlorocyana) Duméril \& Bibron 1837-Dominican Green Anole

A. chlorodius (Deiroptyx chlorodius) Köhler \& Hedges 2016-Pedernales Green Anole

Comment. Nicholson et al. (2018) gave the species name a feminine gender in combination with Deiroptyx, but it is a noun in apposition so it retains the original spelling.

A. christophei (Xiphosurus christophei) Williams 1960-Big-fanned Trunk Anole

A. clivicola Barbour \& Shreve 1935-Turquino Fern Anole

A. coelestinus (Deiroptyx coelestina) Cope 1862-Tiburon Green Anole

A. concolor (Norops concolor) Cope 1962-Isla San Andres Anole

A. confusus (Norops confusus) Estrada \& Garrido 1991—Cabo Cruz Trunk Anole

A. conspersus (Norops conspersus) Garman 1887-Cayman Islands Blue-fanned Anole

Subspecies. Anolis conspersus conspersus (Norops conspersus conspersus) Garman 1887; A. c. lewisi (N. c. lewisi) Grant 1940.

A. cooki (Ctenonotus cooki) Grant 1931-Guanica Pallid Anole

A. cristatellus (Ctenonotus cristatellus) Duméril \& Bibron 1837-Puerto Rican Crested Anole

Subspecies. Anolis cristatellus cristatellus (Ctenonotus cristatellus cristatellus) Duméril \& Bibron 1837; A. c. wileyae (C. c. wileyae) Grant 1931.

A. cupeyalensis Peters 1970-Stripe-bellied Grass Anole

A. cuvieri (Xiphosurus cuvieri) Merrem 1820_Puerto Rican Giant Anole

A. cyanopleurus Cope 1861-Green Fern Anole

Subspecies. Anolis cyanopleurus cyanopleurus Cope 1861; A. c. orientalis Garrido 1975.

A. cyanostictus (Deiroptyx cyanosticta) Mertens 1939-Santo Domingo Green Anole

A. cybotes (Audantia cybotes) Cope 1862-Hispaniolan Stout Anole

Subspecies. Anolis cybotes cybotes (Audantia cybotes cybotes) Cope 1862; A. c. doris (A. c. doris) Barbour 1925; 
A. c. ravifaux (A. c. ravifaux) Schwartz \& Henderson 1982.

A. darlingtoni (Deiroptyx darlingtoni) (Cochran 1935)—La Hotte Twig Anole

A. delafuentei (Norops delafuentei) Garrido 1982-Escambray Crested Anole

A. demissus (Deiroptyx demissa) Schwartz 1969-Cayemite Green Anole

A. desechensis (Ctenonotus desechensis) Heatwole 1976-Desecheo Anole

A. desiradei (Ctenonotus desiradei) Lazell 1964-La Desirade Anole

A. distichus (Ctenonotus distichus) Cope 1861-North Caribbean Bark Anole

Subspecies. Anolis distichus distichus (Ctenonotus distichus distichus) Cope 1861; A. d. biminiensis (C. d. biminiensis) Oliver 1948; A. d. dapsilis (C. d. dapsilis) Schwartz 1968; A. d. distichoides (C. d. distichoides) Rosén 1911; A. d. dominicensis (C. d. dominicensis) Reinhardt \& Lütken 1863; A. d. favillarum (C. d. favillarum) Schwartz 1968; A. d. ignigularis (C. d. ignigularis) Mertens 1939; A. d. juliae (C. d. juliae) Cochran 1934; A. d. ocior (C. d. ocior) Schwartz 1968; A. d. patruelis (C. d. patruelis) Schwartz 1968; A. d. properus (C. d. properus) Schwartz 1968; A. d. ravitergum (C. d. ravitergum) Schwartz 1968; A. d. sejunctus (C. d. sejunctus) Schwartz 1968; A. d. suppar (C. d. suppar) Schwartz 1968; A.d. tostus (C. d. tostus) Schwartz 1968.

Comments. This taxon could well represent a species complex. Glor \& Laport (2012) recommended species-level recognition of five taxa (A. d. dominicensis A. d. favillarum A. d. ignigularis A. d. properus and A. d. ravitergum), and MacGuigan et al. (2017) identified "seven distinct evolutionary lineages still experiencing a limited degree of gene flow" and labeled these as "young lineages at varying stages of divergence." We herein tentatively retain them as subspecies.

A. divius (Deiroptyx divius) Köhler \& Hedges 2016-Baoruco Blue Anole

Comment. Nicholson et al. (2018) gave the species name a feminine gender in combination with Deiroptyx, but it is a noun ("sky god") in apposition so it retains the original spelling.

A. dolichocephalus (Deiroptyx dolichocephala) Williams 1963-La Hotte Long-snouted Anole

Subspecies. Anolis dolichocephalus dolichocephalus (Deiroptyx dolichocephala dolichocephala) Williams 1963; A. d. portusalus (D. d. portusala) Schwartz 1978; A. d. sarmenticola (D. d. sarmenticola) Schwartz 1978.

A. dominicanus (Deiroptyx dominicana) Rieppel 1980-La Toca Anole

Comment. This taxon is known only from a fossil embedded in amber (Rieppel 1980).

A. eladioi (Deiroptyx eladioi) Köhler \& Hedges 2016-Baoruco Green Anole

A. equestris (Deiroptyx equestris) Merrem 1820-Cuban Giant Anole

Subspecies. Anolis equestris equestris (Deiroptyx equestris equestris) Merrem 1820; A. e. brujensis (D. e. brujensis) Garrido 2001; A. e. buidei (D. e. buidei) Schwartz \& Garrido 1972; A. e. cincoleguas (D. e. cincoleguas) Garrido 1981; A. e. cyaneus (D. e. cyanea) Garrido \& Estrada 2001; A. e. juraguensis (D. e. juraguensis) Schwartz \& Garrido 1972; A. e. persparsus (D. e. persparsa) Schwartz \& Garrido 1972; A. e. potior (D. e. potior) Schwartz \& Thomas 1975; A. e. sabinalensis (D. e. sabinalensis) Garrido \& Moreno 2001; A. e. thomasi (D. e. thomasi) Schwartz 1958; $A$. e. verreonensis (D. e. verreonensis) Schwartz \& Garrido 1972.

A. ernestwilliamsi (Ctenonotus ernestwilliamsi) Lazell 1983-Carrot Rock Anole

A. etheridgei Williams 1962 (Chamaelinorops darlingtoni (Cochran 1939))—Montane Bush Anole

Comment. Anolis etheridgei replaced Anolis darlingtoni Cochran 1939 to avoid homonyny with Anolis darlingtoni (Cochran 1935). If different genera are recognized, that action reverts and therefore Anolis etheridgei becomes a synonym of Chamaelinorops darlingtoni.

A. eugenegrahami (Xiphosurus eugenegrahami) Schwartz 1978-Black Stream Anole

A. evermanni (Ctenonotus evermanni) Stejneger 1904-Puerto Rican Emerald Anole

A. extremus (Dactyloa extrema) Garman 1887-Barbados Anole

A. fairchildi Barbour \& Shreve 1935—Cay Sal Anole

A. ferreus (Ctenonotus ferreus) (Cope 1864)—Marie-Galante Anole

A. fowleri (Chamaelinorops fowleri) Schwartz 1973-Green-banded Anole 
A. fugitivus Garrido 1975-Green-headed Grass Anole

A. garmani (Norops garmani) Stejneger 1899-Jamaican Giant Anole

A. garridoi Díaz, Estrada, \& Moreno 1996-Escambray Twig Anole

A. gingivinus (Ctenonotus gingivinus) Cope 1864-Anguilla Bank Tree Anole

A. gonavensis (Deiroptyx gonavensis) Köhler \& Hedges 2016-Gonave Twig Anole

A. grahami (Norops grahami) Gray 1845-Jamaican Turquoise Anole

Subspecies. Anolis grahami grahami (Norops grahami grahami) Gray 1845; A. g. aquarum (N. g. aquarum) Underwood \& Williams 1959.

A. griseus (Dactyloa grisea) Garman 1887-Saint Vincent Tree Anole

A. guafe (Norops guafe) Estrada \& Garrido 1991—Cabo Cruz Banded Anole

A. guamuhaya (Xiphosurus guamuhaya) (Garrido, Pérez-Beato, \& Moreno 1991)—Escambray Bearded Anole

A. guazuma Garrido 1984-Turquino Twig Anole

A. gundlachi (Ctenonotus gundlachi) Peters 1876-Yellow-chinned Anole

A. haetianus (Audantia haetiana) Garman 1887-Tiburon Stout Anole

A. hendersoni (Deiroptyx hendersoni) Cochran 1923-La Selle Long-snouted Anole

Subspecies. Anolis hendersoni hendersoni (Deiroptyx hendersoni hendersoni) Cochran 1923; A. h. ravidormitans (D. h. ravidormitans) Schwartz 1978.

A. homolechis (Norops homolechis) Cope 1864-Cuban White-fanned Anole

Subspecies. Anolis homolechis homolechis (Norops homolechis homolechis) Cope 1864; A. h. turquinensis (N. h. turquinensis) Garrido 1973.

A. imias (Norops imias) Ruibal \& Williams 1961—Imias Rock Anole

A. incredulus Garrido \& Moreno 1999-Turquino Emerald Anole

A. inexpectatus Garrido \& Estrada 1989—Pineland Bush Anole

A. insolitus (Chamaelinorops insolitus) Williams \& Rand 1969-Cordillera Central Twig Anole

A. isolepis (Cope 1861)—Dwarf Green Anole

A. juangundlachi Garrido 1975-Yellow-lipped Grass Anole

A. jubar (Norops jubar) Schwartz 1968-Cuban Coast Anole

Subspecies. Anolis jubar jubar (Norops jubar jubar) Schwartz 1968; A. j. albertschwartzi (N. j. albertschwartzi) Garrido 1973; A. j. balaenarum (N. j. balaenarium) Schwartz 1968; A. j. cocoensis (N. j. cocoensis) Estrada \& Garrido 1990; A. j. cuneus (N. j. cuneus) Schwartz 1968; A. j. gibarensis (N. j. gibarensis) Garrido 1973; A. j. maisiensis (N.j. maisiensis) Garrido 1973; A. j. oriens (N. j. oriens) Schwartz 1968; A. j. santamariae (N. j. santamariae) Garrido 1973; A. j. yaguajayensis (N. j. yaguajayensis) Garrido 1973.

A. koopmani (Deiroptyx koopmani) Rand 1961-Haitian Brown Red-bellied Anole

A. krugi (Ctenonotus krugi) Peters 1877 ("1876")—Olive Bush Anole

A. landestoyi (Xiphosurus landestoyi) Mahler, Lambert, Geneva, Ng, Hedges, Losos, \& Glor 2016—Hispaniolan Chamaeleon Anole

A. leachii (Ctenonotus leachii) Duméril \& Bibron 1837-Antigua Bank Tree Anole

A. leucodera (Deiroptyx leucodera) Köhler \& Hedges 2016-Bombardopolis Green Anole

A. lineatopus (Norops lineatopus) Gray 1840-Stripe-footed Anole

Subspecies. Anolis lineatopus lineatopus (Norops lineatopus lineatopus) Gray 1840; A. I. ahenobarbus (N. I. ahenobarbus) Underwood \& Williams 1959; A. I. merope (N. I. merope) Underwood \& Williams 1959; A. I. neckeri Grant 1940 (N. I. neckeri).

A. lineatus (Norops lineatus) Daudin 1802-Curacao Striped Anole

A. litoralis Garrido 1975-Oriente Pallid Anole

A. lividus (Ctenonotus lividus) Garman 1887-Montserrat Anole

A. longiceps Schmidt 1919-Navassa Anole 


\section{A. longitibialis (Audantia longitibialis) Noble 1923-Barahona Stout Anole}

Subspecies. Anolis longitibialis longitibialis (Audantia longitibialis longitibialis) Noble 1923; A. I. specuum (A. I. specuum) Schwartz 1979.

A. loysiana Duméril \& Bibron 1837-Spiny Anole

A. luciae (Dactyloa luciae) Garman 1887-Saint Lucia Anole

A. lucius Duméril \& Bibron 1837-Slender Cliff Anole

A. luteogularis (Deiroptyx luteogularis) Noble \& Hassler 1935-Western Giant Anole

Subspecies. Anolis luteogularis luteogularis (Deiroptyx luteogularis luteogularis) Noble \& Hassler 1935; A. I. calceus (D. I. calcea) Schwartz \& Garrido 1972; A. I. coctilis (D. I. coctilis) Schwartz \& Garrido 1972; A. I. delacruzi (D. I. delacruzi) Schwartz \& Garrido 1972; A. I. hassleri (D. I. hasserli) Barbour \& Shreve 1935; A. I. jaumei (D. I. jaumei) Schwartz \& Garrido 1972; A. I. nivevultus (D. I. nivevulta) Schwartz \& Garrido 1972; A. I. sanfelipensis (D. I. sanfelipensis) Garrido 1975; A. I. sectilis (D. I. sectilis) Schwartz \& Garrido 1972.

A. luteosignifer (Norops luteosignifer) Garman 1888-Cayman Brac Anole

A. macilentus Garrido \& Hedges 1992—Black-cheeked Bush Anole

A. marcanoi (Audantia marcanoi) Williams 1975-Red-fanned Stout Anole

A. marmoratus (Ctenonotus marmoratus) Duméril \& Bibron 1837-Guadeloupe Anole

Subspecies. Anolis marmoratus marmoratus (Ctenonotus marmoratus marmoratus) Duméril \& Bibron 1837; A. m. alliaceus (C. m. alliaceus) Cope 1864; A. m. caryae (C. m. caryae) Lazell 1964; A. m. chrysops (C. m. chrysops) Lazell 1964; A. m. girafus (C. m. girafus) Lazell 1964; A. m. inornatus (C. m. inornatus) Lazell 1964; A. m. kahouannensis (C. m. kahouannensis) Lazell 1964; A. m. setosus (C. m. setosus) Lazell 1964; A. m. speciosus (C. m. speciosus) (Garman 1887).

Comment. The currently recognized subspecies might represent only ecotypes (e.g., Thorpe et al. 2015; Thorpe et al. 2018) and might not warrant taxonomic recognition. We herein tentatively retain them.

A. marron (Ctenonotus marron) Arnold 1980-Jacmel Gracile Anole

A. maynardii Garman 1888-Little Cayman Green Anole

A. mestrei (Norops mestrei) Barbour \& Ramsden 1916—Red-fanned Rock Anole

A. monensis (Ctenonotus monensis) Stejneger 1904-Mona Anole

A. monticola (Deiroptyx monticola) Shreve 1936-La Hotte Bush Anole

Subspecies. Anolis monticola monticola (Deiroptyx monticola monticola) Shreve 1936; A. m. quadrisartus (D. m. quadrisarta) Thomas \& Schwartz 1967.

A. nelsoni (Norops nelsoni) Barbour 1914-Swan Islands Anole

A. noblei (Deiroptyx noblei) Barbour \& Shreve 1935-Oriente Giant Anole

A. nubilus (Ctenonotus nubilus) Garman 1887-Redonda Anole

A. occultus (Deiroptyx occulta) Williams \& Rivero 1965-Puerto Rican Twig Anole

A. oculatus (Ctenonotus oculatus) (Cope 1879)—Dominica Anole

Subspecies. Anolis oculatus oculatus (Ctenonotus oculatus oculatus) (Cope 1879); A. o. cabritensis (C. o. cabritensis) Lazell 1962; A. o. montanus (C. o. montanus) Lazell 1962; A. o. winstoni (C. o. winstoni) Lazell 1962.

Comment. The currently recognized subspecies might represent only ecotypes (e.g., Malhotra \& Thorpe 1994, 1997; Thorpe et al. 2015) and might not warrant taxonomic recognition. We herein tentatively retain them.

A. olssoni (Chamaelinorops olssoni) Schmidt 1919-Desert Grass Anole

Subspecies. Anolis olssoni olssoni (Chamaelinorops olssoni olssoni) Schmidt 1919; A. o. alienus (C. o. alienus) Schwartz 1981; A. o. dominigensis (C. o. dominigensis) Schwartz 1981; A. o. extentus (C. o. extentus) Schwartz 1981; A. o. ferrugicauda (C. o. ferrugicauda) Schwartz 1981; A. o. insulanus (C. o. insulanus) Schwartz 1981; A. o. montivagus (C. o. montivagus) Schwartz 1981; A. o. palloris (C. o. palloris) Schwartz 1981.

A. onca (Norops onca) (O'Shaughnessy 1875)—Bulky Anole

A. opalinus (Norops opalinus) Gosse 1850—Jamaican Opal-bellied Anole 
A. ophiolepis (Norops ophiolepis) Cope 1861-Five-striped Grass Anole

A. oporinus Garrido \& Hedges 2001—Pimienta Green Anole

A. paternus Hardy 1967-Ashy Bush Anole

Subspecies. Anolis paternus paternus Hardy 1967; A. p. pinarensis Garrido 1975.

A. pecuarius (Deiroptyx pecuarius) Schwartz 1969-Ile-a-Vache Green Anole

A. peynadoi (Deiroptyx peynadoi) Mertens 1939-Western Hispaniola Green Anole

A. pigmaequestris (Deiroptyx pigmaequestris) Garrido 1975-Pygmy Giant Anole

A. pinchoti (Norops pinchoti) Cochran 1931—Crab Cay Anole

A. placidus Hedges \& Thomas 1989-Neiba Twig Anole

A. planiceps (Norops planiceps) Troschel 1848-Golden-scaled Anole

A. pogus (Ctenonotus pogus) Lazell 1972-Anguilla Bank Bush Anole

A. poncensis (Ctenonotus poncensis) Stejneger 1904-Ponce Small-fanned Anole

A. porcatus Gray 1840-Cuban Green Anole

Subspecies. Anolis porcatus porcatus Gray 1840; A. p. aracelyae Pérez-Beato 1996.

A. porcus (Xiphosurus porcus) (Cope 1864)—Oriente Bearded Anole

A. prasinorius (Deiroptyx prasinorius) Köhler \& Hedges 2016-Baoruco Green Twig Anole

Comment. Nicholson et al. (2018) gave the species name a feminine gender in combination with Deiroptyx, but it is a noun in apposition so it retains the original spelling.

A. pulchellus (Ctenonotus pulchellus) Duméril \& Bibron 1837—Puerto Rican Bush Anole

A. pumilus Garrido 1988-Cuban Spiny-plant Anole

A. quadriocellifer (Norops quadriocellifer) Barbour \& Ramsden 1919-Cuban Eyespot Anole

A. reconditus (Norops reconditus) Underwood \& Williams 1959—Blue Mountains Anole

A. rejectus Garrido \& Schwartz 1972—Santiago Grass Anole

A. richardii (Dactyloa richardii) Duméril \& Bibron 1837-Grenada Tree Anole

A. ricordii (Xiphosurus ricordii) Duméril \& Bibron 1837-Haitian Giant Anole

Subspecies. Anolis ricordii ricordii (Xiphosurus ricordii ricordii) Duméril \& Bibron 1837; A. r. leberi (X. r. leberi) Williams 1965; A. r. subsolanus (X. r. subsolanis) Schwartz 1974; A. r. viculus (X. r. viculus) Schwartz 1974.

A. rimarum (Deiroptyx rimarum) Thomas \& Schwartz 1967-Artibonite Bush Anole

A. roatanensis (Norops roatanensis) (Köhler \& McCranie 2001)—Roatan Anole

A. rodriguezii (Norops rodriguezii) Bocourt 1873-Middle American Smooth Anole

A. roosevelti (Xiphosurus roosevelti) Grant 1931-Virgin Islands Giant Anole

Comment. This species is almost certainly extinct and has not been collected since 1932 (e.g., de Queiroz \& Mayer 2011; Revell et al. 2015).

A. roquet (Dactyloa roquet) (Bonnaterre 1789)—Martinique Anole

Subspecies. Anolis roquet roquet (Dactyloa roquet roquet) (Bonnaterre 1789); A. r. caracoli (D. r. caracoli) Lazell 1972; A. r. majolgris (D. r. majolgris) Lazell 1972; A. r. salinei (D. r. salinei) Lazell 1972; A. r. summus (D. r. summa) Lazell 1972; A. r. zebrilus (D. r. zebrila) Lazell 1972.

Comment. The currently recognized subspecies might represent ecotypes (e.g., Thorpe et al. 2010) and might not warrant taxonomic recognition. We herein tentatively retain them.

A. rubribarbus (Norops rubribarbus) Barbour \& Ramsden 1919-Cuban Tiger Anole

A. ruibali Navarro \& Garrido 2004-Cabo Cruz Pallid Anole

A. rupinae (Deiroptyx rupinae) Williams \& Webster 1974-Haitian Banded Red-bellied Anole

A. sabanus (Ctenonotus sabanus) Garman 1887-Saba Anole

A. sagrei (Norops sagrei) Cocteau in Duméril \& Bibron 1837-Cuban Brown Anole

Subspecies. Anolis sagrei sagrei (Norops sagrei sagrei) Cocteau in Duméril \& Bibron 1837; A. s. mayensis (N. s. mayensis) Smith \& Burger 1949. 
A. schwartzi (Ctenonotus schwartzi) Lazell 1972-Saint Kitts Bank Bush Anole

A. scriptus (Ctenonotus scriptus) Garman 1887-Southern Bahamas Anole

Subspecies. Anolis scriptus scriptus (Ctenonotus scriptus scriptus) Garman 1887; A. s. leucophaeus (C. s. leucophaeus) Garman 1888; A. s. mariguanae (C. s. mariguanae) Cochran 1931; A. s. sularum (C. s. sularum) Barbour \& Shreve 1935.

A. semilineatus (Chamaelinorops semilineatus) Cope 1864-Hispaniolan Grass Anole

A. sheplani Schwartz 1974-Baoruco Gray Twig Anole

A. shrevei (Audantia shrevei) (Cochran 1939)—Cordillera Central Stout Anole

A. sierramaestrae (Xiphosurus sierramaestrae) Holáňová, Rehák, \& Frynta 2012-Sierra Maestrae Bearded Anole

A. singularis (Deiroptyx singularis) Williams 1965-Macaya Green Twig Anole

A. smallwoodi (Deiroptyx smallwoodi) Schwartz 1964-Green-blotched Giant Anole

Subspecies. Anolis smallwoodi smallwoodi (Deiroptyx smallwoodi smallwoodi) Schwartz 1964; A. s. palardis (D. s. palardis) Schwartz 1964; A. s. saxuliceps (D. s. saxuliceps) Schwartz 1964.

A. smaragdinus Barbour \& Shreve 1935-Bahamian Green Anole

Subspecies. Anolis smaragdinus smaragdinus Barbour \& Shreve 1935; A. s. lerneri Oliver 1948.

A. spectrum Peters 1863-Black-shouldered Ground Anole

A. strahmi (Audantia strahmi) Schwartz 1979-Baoruco Stout Anole

Subspecies. Anolis strahmi strahmi (Audantia strahmi strahmi) Schwartz 1979; A. s. abditus (A. s. abdita) Schwartz 1979.

Comment. Nicholson et al. (2018) gave the subspecies name Audantia strahmi abditus a masculine gender in combination with Audantia, but it is an adjective so it requires the feminine gender.

A. stratulus (Ctenonotus stratulus) Cope 1861-Puerto Rican Spotted Anole

A. terraealtae (Ctenonotus terraealtae) Barbour 1915-Les Saintes Anole

A. terueli Navarro, Fernández, \& Garrido 2001-Yellow-fanned Pallid Anole

A. toldo Fong \& Garrido 2000-Gray-banded Green Anole

A. trinitatis (Dactyloa trinitatis) Reinhardt \& Lütken 1862-Saint Vincent Bush Anole

A. unilobatus (Norops unilobatus) Köhler \& Vesely 2010-Blue-spotted Fan Anole

A. utilensis (Norops utilensis) (Köhler 1996)—Utila Anole

A. valencienni (Norops valencienni) Duméril \& Bibron 1837-Jamaican Twig Anole

A. vanidicus Garrido \& Schwartz 1972-Escambray Grass Anole

A. vermiculatus (Deiroptyx vermiculata) Cocteau in Duméril \& Bibron 1837-Cuban Stream Anole

A. vescus Garrido \& Hedges 1992-Sierra Del Purial Bush Anole

A. villai (Norops villai) Fitch \& Henderson 1996-Great Corn Island Anole

A. viridius (Deiroptyx viridius) Köhler \& Hedges 2016-Barahona Green Anole

Comment. Nicholson et al. (2018) gave the species name a feminine gender in combination with Deiroptyx, but it is a noun in apposition so it retains the original spelling.

A. wattsi (Ctenonotus wattsi) Boulenger 1894-Antigua Bank Bush Anole

A. websteri (Ctenonotus websteri) Arnold 1980-Yellow-bellied Desert Anole

A. whitemani (Audantia whitemani) Williams 1963-Pallid Stout Anole

Subspecies. Anolis whitemani whitemani (Audantia whitemani whitemani) Williams 1963; A. w. lapidosus (A. w. lapidosa) Schwartz 1980.

\section{Family Eublepharidae Boulenger 1883-Eyelid Geckos}

\section{Coleonyx Gray 1845-Banded Geckos}

C. mitratus (Peters 1863)—Central American Banded Gecko 


\section{Family Gekkonidae Gray 1825-Typical Geckos}

\section{Gekko Laurenti 1768-Calling Geckos}

G. gecko (Linnaeus 1758)_Tokay Gecko Subspecies. Gekko gecko gecko (Linnaeus 1758).

\section{Hemidactylus Oken 1817-House Geckos}

Hemidactylus angulatus Hallowell 1854-West African House Gecko

Comment. Genetic data showed that this taxon was introduced on Caribbean islands, probably during the slave-trade period (Weiss \& Hedges 2007), leading those authors to synonymize H. haitianus Meerwarth 1901 with $H$. angulatus. Although future research may show that $H$. angulatus is a complex of species, one of which may take the name $H$. haitianus, current taxonomic usage must reflect published evidence and therefore we do not herein recognize $H$. haitianus.

Hemidactylus frenatus Duméril \& Bibron 1836-Common House Gecko

Hemidactylus garnotii Duméril \& Bibron 1836-Indopacific Gecko

Hemidactylus mabouia (Moreau de Jonnès 1818)_Tropical House Gecko

Hemidactylus palaichthus Kluge 1969-Spiny House Gecko

Hemidactylus turcicus (Linnaeus 1758)—Mediterranean Gecko

\section{Lepidodactylus Fitzinger 1843-Scaly-toed Geckos}

L. lugubris (Duméril \& Bibron 1836)—Mourning Gecko

\section{Family Gymnophthalmidae Merrem 1820—Spectacled Lizards}

\section{Bachia Gray 1845-Bachias}

B. alleni (Barbour 1914)—Antillean Bachia

Comment. This taxon is sometimes considered a subspecies of B. heteropa (e.g., Uetz et al. 2018).

B. flavescens (Bonnaterre 1789) - Yellow-lined Bachia

B. trinitatis (Barbour 1914) - Trinidad Bachia

Comments. Galis et al. (2010) and Kohlsdorf et al. (2010) presented phylogenies suggesting that $B$. heteropa as currently defined is polyphyletic, within a complex including $B$. trinitatis. Bachia alleni is sometimes considered a subspecies of B. heteropa (e.g., John et al. 2012; Uetz et al. 2018).

\section{Gymnophthalmus Merrem 1820-Typical Tegulets}

G. lineatus (Linnaeus 1758)-Striped Tegulet

G. pleii Bocourt 1881-Rough-scaled Tegulet

G. speciosus (Hallowell 1861)—Golden Tegulet

G. underwoodi Grant 1958-Smooth-scaled Tegulet

Comments. This parthenogenetic taxon as currently defined undoubtedly represents a complex of species, all probably of hybrid origins but not necessarily from the same parental stocks (e.g., Williamson \& Powell 2004).

Oreosaurus Peters 1862-Montane Tegulets

O. shrevei (Parker 1935)—Luminous Lizard

Tretioscincus Cope 1862-Smooth Tegulets

T. bifasciatus (Duméril in Duméril \& Bibron 1851)—Rio Magdalena Minitegu

Family Iguanidae Oppel 1811-Iguanas

\section{Ctenosaura Wiegmann 1828-Spiny-tailed Iguanas}


C. bakeri Stejneger 1901—Utila Spiny-tailed Iguana

C. oedirhina de Queiroz 1987-Roatan Spiny-tailed Iguana

C. similis (Gray 1830)—Common Spiny-tailed Iguana

Subspecies. Ctenosaura similis similis (Gray 1831 ["1930"]); C. s. multipunctata Barbour \& Shreve 1934.

\section{Cyclura Harlan 1825-West Indian Rock Iguanas}

Comment. Fossil material, identified as Cyclura sp., is known from New Providence Island, Bahamas (Etheridge 1965; Pregill 1982).

C. carinata Harlan 1824-Turks \& Caicos Iguana

Comment. The taxon C. c. bartschi might not be valid (Bryan et al. 2007).

C. caymanensis Barbour \& Noble 1916-Sister Isles Iguana

Comment. This taxon was described a full species but is sometimes considered a subspecies of $C$. nubila (e.g., Iguana Taxonomy Working Group 2016; Uetz et al. 2018).

C. collei Gray 1845-Jamaican Iguana

C. cornuta (Bonnaterre 1789)—Hispaniolan Rhinoceros Iguana

C. cychlura (Cuvier 1829)—Andros Island Iguana

Comment. Cyclura figginsi and C. inornata were described as full species but often are considered subspecies of C. cychlura (e.g., Iguana Taxonomy Working Group 2016; Uetz et al. 2018).

C. figginsi Barbour 1923-Exuma Islands Iguana

Comment. This taxon was described as a full species but is sometimes considered a subspecies of $C$. cychlura (e.g., Iguana Taxonomy Working Group 2016; Uetz et al. 2018).

C. inornata Barbour \& Noble 1916-Allen Cays Iguana

Comment. This taxon was described as a full species but is sometimes considered a subspecies of $C$. cychlura (e.g., Iguana Taxonomy Working Group 2016; Uetz et al. 2018).

C. lewisi Grant 1941-Grand Cayman Blue Iguana

C. nubila (Gray 1831)—Cuban Iguana

Comment. Cyclura caymanensis is sometimes considered a subspecies of C. nubila (e.g., Iguana Taxonomy Working Group 2016; Uetz et al. 2018).

C. onchiopsis Cope 1885-Navassa Rhinoceros Iguana

Comments. This taxon almost certainly is extinct (e.g., Thomas 1966; Powell 1999; Iguana Taxonomy Working Group 2016). It was described as a full species but Uetz et al. (2018) listed this taxon as a subspecies of $C$. cornuta.

C. pinguis Barbour 1917-Stout Iguana

C. ricordii (Duméril \& Bibron 1837)—Hispaniolan Banded Iguana

C. rileyi Stejneger 1903-San Salvador Iguana

Comment. Cyclura cristata and C. nuchalis are sometimes considered subspecies of C. rileyi (e.g., Iguana Taxonomy Working Group 2016; Uetz et al. 2018).

C. stejnegeri Barbour \& Noble 1916-Mona Rhinoceros Iguana

\section{Iguana Laurenti 1768-Typical Iguanas}

I. delicatissima Laurenti 1768-Lesser Antillean Iguana

I. iguana (Linnaeus 1758)—Green Iguana

Comment. This widely distributed taxon is almost certainly a complex of species (e.g., Stephen et al. 2013).

\section{Family Leiocephalidae Frost \& Etheridge 1989—Curly-tailed Lizards}

\section{Leiocephalus Gray 1827-Curlytails}


L. anonymous Pregill 1984-Arbonite Curlytail

Comment. This taxon is known only from fossil material (Pregill 1984, 1992).

L. apertosulcus Etheridge 1965-San Rafael Curlytail

Comment. This taxon is known only from fossil material (Etheridge 1965; Pregill 1992).

L. altavelensis Noble \& Hassler 1933-Alto Velo Curlytail

Comment. This taxon is sometimes considered a subspecies of L. barahonensis (e.g., Uetz et al. 2018).

L. barahonensis Schmidt 1921_Orange-bellied Curlytail

Subspecies. Leiocephalus barahonensis barahonensis Schmidt 1921; L. b. aureus Cochran 1934; L. b. beatanus Noble 1923; L. b. oxygaster Schwartz 1967.

L. carinatus Gray 1827-Saw-scaled Curlytail

Subspecies. Leiocephalus carinatus carinatus Gray 1827; L. c. aquarius Schwartz \& Ogren 1956; L. c. armouri Barbour \& Shreve 1935; L. C. cayensis Schwartz 1959; L. c. coryi Schmidt 1936; L. C. granti Rabb 1957; L. c. hodsoni Schmidt 1936; L. c. labrossytus Schwartz 1959; L. c. microcyon Schwartz 1959; L. c. mogotensis Schwartz 1959; L. C. virescens Stenjneger 1901; L. C. zayasi Schwartz 1959.

Comment. This taxon might represent a species complex. Pregill (1992) noted some skeletal differences between some subspecies, but his "samples of these populations were inadequate to assess accurately the significance of these differences."

L. cubensis (Gray 1840)—Cuban Brown Curlytail

Subspecies. Leiocephalus cubensis cubensis (Gray 1840); L. c. gigas Schwartz 1959; L. C. minor Varona \& Garrido 1970; L. C. pambasileus Schwartz 1959; L. c. paraphrus Schwartz 1959.

L. cuneus Etheridge 1964-Leeward Islands Curlytail

Comment. This taxon is known only from fossil material (Etheridge 1964; Pregill 1992).

L. endomychus Schwartz 1967-Hinche Curlytail

L. eremitus Cope 1868-Navassa Curlytail

Comment. This species almost certainly is extinct (Thomas 1966; Pregill 1992; Powell 1999).

L. etheridgei Pregill 1981-Morovis Curlytail

Comment. This taxon is known only from fossil material (Pregill 1981, 1992).

L. greenwayi Barbour \& Shreve 1935-East Plana Curlytail

L. herminieri (Duméril \& Bibron 1837)—Martinique Curlytail

Comment. This species almost certainly is extinct (Barbour 1914; Pregill 1992; Breuil 2002).

L. inaguae Cochran 1931 - Inagua Curlytail

L. jamaicensis Etheridge 1966-Jamaican Curlytail

Comment. This taxon is known only from fossil material (Etheridge 1966; Pregill 1992).

L. loxogrammus Cope 1887-San Salvador Curlytail

Subspecies. Leiocephalus loxogrammus loxogrammus Cope 1887; L. I. parnelli Barbour \& Shreve 1935.

Comment. The recognition of the two subspecies might not be valid (Powell 2018).

L. Iunatus Cochran 1934-Hispaniolan Maskless Curlytail

Subspecies. Leiocephalus lunatus lunatus Cochran 1934; L. I. arenicolor Mertens 1939; L. I. lewisi Schwartz 1967;

L. I. Iouisae Cochran 1934; L. I. melaenoscelis Schwartz 1967; L. I. thomasi Schwartz 1967.

L. macropus Cope 1862-Cuban Side-blotched Curlytail

Subspecies. Leiocephalus macropus macropus Cope 1862; L. m. aegialus Schwartz \& Garrido 1967; L. m. asbolomus Schwartz \& Garrido 1967; L. m. felinoi Garrido 1979; L. m. hoplites Zug 1959; L. m. hyacinthurus Zug 1959; L. m. immaculatus Hardy 1958; L. m. koopmani Zug 1959; L. m. lenticulatus Garrido 1973; L. m. phylax Schwartz \& Garrido 1967; L. m. torrei Garrido 1979.

L. melanochlorus Cope 1862-Tiburon Curlytail

Subspecies. Leiocephalus melanochlorus melanochlorus Cope 1862; L. m. hypsistus Schwartz 1966. 
L. onaneyi Garrido 1973-Guantanamo Striped Curlytail

L. partidus Pregill 1981—Guanica Curlytail

Comment. This taxon is known only from fossil material (Pregill 1981, 1992).

L. personatus Cope 1862-Hispaniolan Masked Curlytail

Subspecies. Leiocephalus personatus personatus Cope 1862; L. p. actites Schwartz 1967; L. p. agraulus Schwartz 1967; L. p. budeni Schwartz 1967; L. p. elattoprosopon Gali Schwartz \& Suarez 1988; L. p. mentalis Cochran 1932;

L. p. poililometes Schwartz 1969; L. p. pyrrholaemus Schwartz 1971; L. p. scalaris Cochran 1932; L. p. socoensis Gali \& Schwartz 1982; L. p. tarachodes Schwartz 1967; L. p. trujilloensis Mertens 1939.

L. pratensis (Cochran 1928)—Haitian Striped Curlytail

Subspecies. Leiocephalus pratensis pratensis (Cochran 1928); L. p. chimarus Schwartz 1979.

L. psammodromus Barbour 1916-Turks \& Caicos Curlytail

Subspecies. Leiocephalus psammodromus psammodromus Barbour 1916; L. p. aphretor Schwartz 1967; L. p. apocrinus Schwartz 1967; L. p. cacodoxus Schwartz 1967; L. p. hyphantus Schwartz 1967; L. p. mounax Schwartz 1967.

L. punctatus Cochran 1931—Crooked-Acklins Curlytail

L. raviceps Cope 1862-Pallid Curlytail

Subspecies. Leiocephalus raviceps raviceps Cope 1862; L. r. delavarai Garrido 1973; L. r. jaumei Schwartz \& Garrido 1968; L. r. klinikowski Schwartz 1960; L. r. uzzelli Schwartz 1960.

L. rhutidira Schwartz 1979-Haitian Black-throated Curlytail

L. schreibersii (Gravenhorst 1837)—Red-sided Curlytail

Subspecies. Leiocephalus schreibersii schreibersii (Gravenhorst 1837); L. s. nesomorus Schwartz 1968.

L. semilineatus Dunn 1920—Hispaniolan Pale-bellied Curlytail

L. sixtoi Köhler, Bobadilla, \& Hedges 2016-Hispaniolan Dune Curlytail

L. stictigaster Schwartz 1959—Cuban Striped Curlytail

Subspecies. Leiocephalus stictigaster stictigaster Schwartz 1959; L. s. astictus Schwartz 1959; L. s. celeustes Schwartz \& Garrido 1968; L. s. exotheotus Schwartz 1959; L. s. gibarensis Schwartz \& Garrido 1968; L. s. lipomator Schwartz \& Garrido 1968; L. s. lucianus Schwartz 1960; L. s. naranjoi Schwartz \& Garrido 1968; L. s. ophiplacodes Schwartz 1964; L. s. parasphex Schwartz 1964; L. s. septentrionalis Garrido 1975; L. s. sierrae Schwartz 1959.

L. varius Garman 1887-Cayman Curlytail

L. vinculum Cochran 1928-Gonave Curlytail

Family Mabuyidae Mittleman 1952-Sun Skinks

\section{Alinea Hedges \& Conn 2012-Caribbean Stripeless Skinks}

A. lanceolata (Cope 1862)—Barbados Skink

A. luciae (Garman 1887)—Saint Lucia Skink

\section{Capitellum Hedges \& Conn 2012-Antillean Small-headed Skinks}

C. mariagalantae Hedges \& Conn 2012-Marie-Galante Skink

C. metallicum (Bocourt 1879)_Lesser Martinique Skink

C. parvicruzae Hedges \& Conn 2012-Lesser Saint Croix Skink

\section{Copeoglossum Tschudi 1845-Neotropical Spotted Skinks}

C. aurae Hedges \& Conn 2012-Greater Windward Skink

C. redondae Hedges \& Conn 2012-Redonda Skink

\section{Mabuya Fitzinger 1826-Antillean Two-lined Skinks}

M. cochonae Hedges \& Conn 2012-Cochons Skink 
M. desiradae Hedges \& Conn 2012-Désirade Skink

M. dominicana (Garman 1887)—Dominica Skink

M. grandisterrae Hedges \& Conn 2012-Grande-Terre Skink

M. guadeloupae Hedges \& Conn 2012-Guadeloupean Skink

M. hispaniolae Hedges and Conn 2012-Hispaniolan Two-lined Skink

M. mabouya (Bonnaterre 1879)—Greater Martinique Skink

M. montserratae Hedges \& Conn 2012-Montserrat Skink

M. parviterrae Hedges, Lorvelec, Barré, Berchel, Combot, Vidal, \& Pavis 2016—Petite Terre Skink

\section{Marisora Hedges \& Conn 2012-Middle American Skinks}

M. aurulae Hedges \& Conn 2012-Lesser Windward Skink

M. berengerae (Miralles 2006) n. comb.-San Andrés Skink

Comment. Although Pinto-Sánchez et al. (2015) placed this taxon in the genus Mabuya after rejecting the genera recognized by Hedges \& Conn (2012), their data indicated that it should be in Marisora instead of Alinea.

M. magnacornae Hedges \& Conn 2012-Corn Island Skink

M. pergravis (Barbour 1921) n. comb.-Providencia Island Skink

Comment. Although Pinto-Sánchez et al. (2015) placed this taxon in the genus Mabuya after rejecting the genera recognized by Hedges \& Conn (2012), their data indicated that it should be in Marisora instead of Alinea.

M. roatanae Hedges \& Conn 2012-Honduran Skink

\section{Spondylurus Fitzinger 1826-Antillean Four-lined Skinks}

S. anegadae Hedges \& Conn 2012-Anegada Skink

S. caicosae Hedges \& Conn 2012-Caicos Islands Skink

S. culebrae Hedges \& Conn 2012-Culebra Skink

S. fulgidus (Cope 1862)—Jamaican Skink

S. haitiae Hedges \& Conn 2012-Hispaniolan Four-lined Skink

S. lineolatus (Noble \& Hassler 1933)—Hispaniolan Ten-lined Skink

S. macleani (Mayer \& Lazell 2000)—Carrot Rock Skink

S. magnacruzae Hedges \& Conn 2012-Greater Saint Croix Skink

S. martinae Hedges \& Conn 2012-Saint Martin Skink

S. monae Hedges \& Conn 2012-Mona Skink

S. monitae Hedges \& Conn 2012-Monito Skink

S. nitidus (Garman 1887)—Puerto Rican Skink

S. powelli Hedges \& Conn 2012-Anguilla Bank Skink

S. semitaeniatus (Wiegmann 1837)—Lesser Virgin Islands Skink

S. sloanii (Daudin 1803)—Virgin Islands Bronze Skink

S. spilonotus (Wiegmann 1837)—Greater Virgin Islands Skink

S. turksae Hedges \& Conn 2012-Turks Islands Skink

Family Phrynosomatidae Fitzinger 1843-Earless, Spiny, Tree, Side-Blotched, Horned Lizards

\section{Sceloporus Dickerson 1919-Spiny Lizards}

S. cozumelae Jones 1927—Cozumel Spiny Lizard

\section{Family Phyllodactylidae Gamble, Bauer, Greenbaum, \& Jackman 2008-Leaf-toed Geckos}




\section{Phyllodactylus Gray 1828-American Leaf-toed Geckos}

P. hispaniolae Schwartz 1980—Dominican Leaf-toed Gecko

P. insularis Dixon 1960—Belize Leaf-toed Gecko

P. julieni Cope 1885-Aruba Leaf-toed Gecko

P. martini Lidth de Jeude 1887-Dutch Leaf-toed Gecko

P. palmeus Dixon 1968-Honduran Leaf-toed Gecko

P. paralepis McCranie \& Hedges 2013-Guanaja Leaf-toed Gecko

P. pulcher Gray 1830—Barbados Leaf-toed Gecko

P. rutteni Hummelinck 1940-Venezuelan Leaf-toed Gecko

P. sommeri Schwartz 1980-Haitian Leaf-toed Gecko

P. tuberculosis Wiegmann in Meyen 1834-Yellow-bellied Leaf-toed Gecko

Subspecies. Phyllodactylus tuberculosis ingeri Dixon 1964.

P. wirshingi Kerster \& Smith 1955—Puerto Rican Leaf-toed Gecko

\section{Tarentola Gray 1825-Wall Geckos}

T. albertschwartzi Sprackland \& Swinney 1998-Jamaican Giant Gecko

Comment. This taxon likely is extinct (Wilson \& Hedges 2016).

T. americana (Gray 1831)_American Wall Gecko

Subspecies. Tarentola americana americana (Gray 1831); T. a. warreni Schwartz 1968.

T. crombiei Díaz \& Hedges 2008-Oriente Tuberculate Gecko

\section{Thecadactylus Oken 1817-Turnip-tailed Geckos}

T. oskrobapreinorum Köhler \& Vesely 2011-Saint Martin Turnip-tailed Gecko

T. rapicauda (Houttuyn 1782)—Northern Turnip-tailed Gecko

\section{Family Polychrotidae Fitzinger 1843-Bush Anoles}

\section{Polychrus Cuvier 1817-Bush Anoles}

P. auduboni (Hallowell 1846 ["1845"])—Smooth-backed Bush Anole

\section{Family Scincidae Gray 1825-Typical Skinks}

\section{Mesoscincus Griffith, Ngo, \& Murphy 2000—Central American Skinks}

M. schwartzei (Fischer 1884)—Mayan Black-headed Skink

\section{Family Sphaerodactylidae Underwood 1954-Dwarf Geckos}

\section{Aristelliger Cope 1862-Croaking Geckos}

Comment. Pregill (1982) referred fossil material from New Providence (Bahamas) to Aristelliger sp.; no species of Aristelliger currently inhabits the Great Bahama Bank.

A. barbouri Noble \& Klingel 1932 — Inagua Croaking Gecko

A. cochranae Grant 1931-Navassa Croaking Gecko

A. expectatus Cochran 1933-Hispaniolan Desert Croaking Gecko

A. georgeensis (Bocourt 1873)—Saint George Island Croaking Gecko

A. hechti Schwartz \& Crombie 1975-Caicos Croaking Gecko

A. lar Cope 1861 (1862)—Hispaniolan Giant Croaking Gecko

A. nelsoni Barbour 1914-Swan Islands Croaking Gecko

Comment. This taxon is sometimes considered a subspecies of A. praesignis (e.g., Uetz et al. 2018). 


\section{A. praesignis (Hallowell 1857)—Jamaican Croaking Gecko}

Comment. Aristelliger nelsoni is sometimes considered a subspecies of this taxon (e.g., Uetz et al. 2018).

A. reyesi Díaz \& Hedges 2009-Cuban Croaking Gecko

\section{Gonatodes Fitzinger 1843-Clawed Geckos}

G. albogularis (Duméril \& Bibron 1836)—White-throated Clawed Gecko

Subspecies. Gonatodes albogularis albogularis (Duméril \& Bibron 1836); G. a. fuscus (Hallowell 1855); G. a. notatus (Reinhardt \& Lütken 1862).

G. antillensis (Lidth de Jeude 1887)—Venezuelan Coastal Clawed Gecko

G. ceciliae Donoso-Barros 1966-Brilliant Clawed Gecko

G. daudini Powell \& Henderson 2005-Union Island Clawed Gecko

G. humeralis (Guichenot 1855)—South American Clawed Gecko

G. naufragus Rivas, Ugueto, Schargel, Barros, Velozo, \& Sánchez 2013-La Blanquilla Clawed Gecko

G. ocellatus (Gray 1831)—Eyespot Clawed Gecko

G. taniae Roze 1963-Ring-necked Clawed Gecko

G. vittatus (Lichtenstein 1856)—Striped Clawed Gecko

\section{Sphaerodactylus Wagler 1830-Geckolets}

S. alphus McCranie \& Hedges 2013-Guanaja Large-scaled Geckolet

S. altavelensis Noble \& Hassler 1933-Hispaniolan Desert Geckolet

Subspecies. Sphaerodactylus altavelensis altavelensis Noble \& Hassler 1933; S. a. brevirostratus Shreve 1968; S.

a. enriquilloensis Shreve 1968; S. a. lucioi Thomas \& Schwartz 1983.

S. argivus Garman 1888-Cayman Islands Geckolet

Subspecies. Sphaerodactylus argivus argivus Garman 1888; S. a. bartschi Cochran 1934; S. a. lewisi Grant 1941.

S. argus Gosse 1850—West Caribbean Ocellated Geckolet

Subspecies. Sphaerodactylus argus argus Gosse 1850; S. a. andresensis Dunn \& Saxe 1950.

S. ariasae Hedges \& Thomas 2001-Jaragua Geckolet

S. armasi Schwartz \& Garrido 1974-Guantanamo Coastal Geckolet

S. armstrongi Noble \& Hassler 1933-Southern Forest Geckolet

Subspecies. Sphaerodactylus armstrongi armstrongi Noble \& Hassler 1933; S. a. hypsinephes Thomas \& Schwartz 1983.

S. asterulus Schwartz \& Graham 1980—Haitian Stippled Geckolet

S. beattyi Grant 1937-Saint Croix Geckolet

Subspecies. Sphaerodactylus beattyi beattyi Grant 1937; S. b. seamani Thomas \& Schwartz 1966.

S. becki Schmidt 1919-Navassa Geckolet

S. bromeliarum Peters \& Schwartz 1972-Cuban Bromeliad Geckolet

S. caicosensis Cochran 1934-Caicos Geckolet

S. callocricus Schwartz 1976-Rough-banded Geckolet

S. celicara Garrido \& Schwartz 1982-Baracoan Eyespot Geckolet

S. ciguapa Daza \& Bauer 2012-Santiago Geckolet

Comment. This species is known only from a single fossil specimen embedded in amber (Daza \& Bauer 2012).

S. cinereus Wagler 1830-Haitian Broad-banded Geckolet

Subspecies. Sphaerodactylus cinereus cinereus Wagler 1830; S. c. stejnegeri Cochran 1931.

S. clenchi Shreve 1968-Orange-spotted Geckolet

Subspecies. Sphaerodactylus clenchi clenchi Shreve 1968; S. c. apocoptus Schwartz 1983.

S. cochranae Ruibal 1946-Haitises Striped Geckolet

S. continentalis Werner 1896-Upper Central American Geckolet 
S. copei Steindachner 1867-Haitian Big-scaled Geckolet

Subspecies. Sphaerodactylus copei copei Steindachner 1867; S. c. astreptus Schwartz 1975; S. c. cataplexis Schwartz \& Thomas 1965; S. c. deuterus Schwartz 1975; S. c. enochrus Schwartz \& Thomas 1965; S. c. pelates Schwartz 1975; S. c. picturatus Garman 1887; S. c. polyommatus Thomas 1968; S. c. websteri Schwartz 1975.

S. corticola Garman 1888-Central Bahamas Geckolet

Subspecies. Sphaerodactylus corticola corticola Garman 1888; S. c. aporrox Schwartz 1968; S. c. campter Schwartz 1968; S. c. soter Schwartz 1968.

S. cricoderus Thomas, Hedges, \& Garrido 1992-Turquino Collared Geckolet

S. cryphius Thomas \& Schwartz 1977—Desert Pygmy Geckolet

S. dacnicolor Barbour 1910—Jamaican Tailspotted Geckolet

S. darlingtoni Shreve 1968-Northern Forest Geckolet

Subspecies. Sphaerodactylus darlingtoni darlingtoni Shreve 1968; S. d. bobilini Thomas \& Schwartz 1983; S. d. mekistus Thomas \& Schwartz 1983; S. d. noblei Shreve 1968.

S. difficilis Barbour 1914-Hispaniolan Eyespotted Geckolet

Subspecies. Sphaerodactylus difficilis difficilis Barbour 1914; S. d. anthracomus Schwartz 1983; S. d. diolenius Schwartz 1983; S. d. euopter Schwartz 1983; S. d. lycauges Schwartz 1983; S. d. peratus Schwartz 1983; S. d. typhlopous Schwartz 1983.

S. dimorphicus Fong \& Díaz 2004-Santiago De Cuba Geckolet

S. docimus Schwartz \& Garrido 1985-Oriente Soft-banded Geckolet

S. dommeli Böhme 1984-La Toca Geckolet

Comment. This species is known only from a single fossil specimen embedded in amber (Böhme 1984; Daza et al. 2012).

S. elasmorhynchus Thomas 1966-Shield-snouted Geckolet

S. elegans (MacCleay 1834)—Ashy Geckolet

Subspecies. Sphaerodactylus elegans elegans (MacCleay 1834); S. e. punctatissimus Duméril \& Bibron 1836.

S. elegantulus Barbour 1917-Antigua Bank Geckolet

S. epiurus Thomas \& Hedges 1993-Hispaniolan Tailspot Geckolet

S. exsul Barbour 1914-Swan Islands Geckolet

S. fantasticus Duméril \& Bibron 1836-Southern Leeward Geckolet

Subspecies. Sphaerodactylus fantasticus fantasticus Duméril \& Bibron 1836; S. f. anidrotus Thomas 1964; S. f. fuga Thomas 1964; S. f. hippomanes Thomas 1964; S. f. karukera Thomas 1964; S. f. ligniservulus King 1962; S. f. orescius Thomas 1964; S. f. tartaropylorus Thomas 1964.

S. festus Barbour 1915-Western Martinique Geckolet

Comment. This taxon is sometimes considered a subspecies of S. vincenti (e.g., Uetz et al. 2018).

S. gaigeae Grant 1932-Chevronate Geckolet

S. gilvitorques Cope 1862-Jamaican Collared Geckolet

S. glaucus Cope 1866-Central American Collared Geckolet

S. goniorhynchus Cope 1895-Jamaican Forest Geckolet

S. guanajae McCranie \& Hedges 2012-Guanaja Head-spotted Geckolet

S. inaguae Noble \& Klingel 1932-Inagua Geckolet

S. intermedius Barbour \& Ramsden 1919-North Coast Banded Geckolet

S. klauberi Grant 1931—Puerto Rican Upland Geckolet

S. ladae Thomas \& Hedges 1988-Spotted Agave Geckolet

S. lazelli Shreve 1968-Cap-Haitien Geckolet

S. leonardovaldesi McCranie \& Hedges 2012-Roatan Small-scaled Geckolet

S. leucaster Schwartz 1973-Broken-striped Geckolet 
S. levinsi Heatwole 1968-Desecheo Geckolet

S. macrolepis Günther 1859-Puerto Rican Eyespotted Geckolet

Subspecies. Sphaerodactylus macrolepis macrolepis Günther 1859; S. m. ateles Thomas \& Schwartz 1966; S. m. grandisquamis Stejneger 1904; S. m. guarionex Thomas \& Schwartz 1966; S. m. inigoi Thomas \& Schwartz 1966; S. m. mimetes Thomas \& Schwartz 1966; S. m. phoberus Thomas \& Schwartz 1966; S. m. spanius Thomas \& Schwartz 1966; S. m. stibarus Thomas \& Schwartz 1966.

S. mariguanae Cochran 1934-South Bahamian Geckolet

S. microlepis Reinhardt \& Lütken 1862-Saint Lucia Geckolet

Subspecies. Sphaerodactylus microlepis microlepis Reinhardt \& Lütken 1862; S. m. thomasi Schwartz 1965.

S. micropithecus Schwartz 1977-Monito Geckolet

S. millepunctatus Hallowell 1861_Lower Central American Geckolet

S. molei Boettger 1894-Tobago Least Geckolet

S. monensis Meerwarth 1901-Mona Geckolet

S. nicholsi Grant 1931—Puerto Rican Crescent Geckolet

S. nigropunctatus Gray 1845-Three-banded Geckolet

Subspecies. Sphaerodactylus nigropunctatus nigropunctatus Gray 1845; S. n. alayoi Grant 1959; S. n. atessares Thomas \& Schwartz 1966; S. n. decoratus Garman 1888; S. n. flavicauda Barbour 1904; S. n. gibbus Barbour 1921; S. n. granti Thomas \& Schwartz 1966; S. n. lissodesmus Thomas \& Schwartz 1966; S. n. ocujal Thomas \& Schwartz 1966; S. n. porrasi Schwartz 1972; S. n. strategus Thomas \& Schwartz 1966.

S. notatus (Baird 1858)—Brown-speckled Geckolet

Subspecies. Sphaerodactylus notatus notatus (Baird 1858); S. n. amaurus Schwartz 1966; S. n. atactus Schwartz 1966; S. n. peltastes Schwartz 1966.

S. nycteropus Thomas \& Schwartz 1977—Haitian Flat-headed Geckolet

S. ocoae Schwartz \& Thomas 1977-Ocoa Geckolet

S. oliveri Grant 1944-Escambray Spotted Geckolet

S. omoglaux Thomas 1982-Haitian Border Geckolet

S. oxyrhinus Gosse 1850-Jamaican Sharp-nosed Geckolet

S. parkeri (Grant 1939)—South Jamaican Banded Geckolet

S. parthenopion Thomas 1965-Virgin Islands Dwarf Geckolet

S. parvus King 1962-Anguilla Bank Geckolet

S. perissodactylius Thomas \& Hedges 1988-Martin Garcia Geckolet

S. phyzacinus Thomas 1965-Les Saintes Geckolet

S. pimienta Thomas, Hedges, \& Garrido 1998-Cuban Pepper Geckolet

S. plummeri Thomas \& Hedges 1992-Barahona Big-scaled Geckolet

S. poindexteri McCranie \& Hedges 2013-Utila Small-scaled Geckolet

S. ramsdeni Ruibal 1959-Cuban Forest Geckolet

S. randi Shreve 1968-Barahona Speckled Geckolet

Subspecies. Sphaerodactylus randi randi Shreve 1968; S. r. methorius Schwartz 1977; S. r. strahmi Schwartz 1977.

S. rhabdotus Schwartz 1970—Hispaniolan Two-striped Geckolet

S. richardi Hedges \& Garrido 1993-Zapata Big-scaled Geckolet

S. richardsonii Gray 1845—Northern Jamaica Banded Geckolet

Subspecies. Sphaerodactylus richardsonii richardsonii Gray 1845; S. r. gossei Grant 1939.

S. roosevelti Grant 1931—Guanica Khaki Geckolet

S. rosaurae Parker 1940-Bay Islands Large-scaled Geckolet

S. ruibali Grant 1959—Guantanamo Bay Geckolet 
S. sabanus Cochran 1938-Saint Kitts Bank Geckolet

S. samanensis Cochran 1932-Haitises Banded Geckolet

S. savagei Shreve 1968-Altagracia Speckled Geckolet

Subspecies. Sphaerodactylus savagei savagei Shreve 1968; S. s. juanilloensis Shreve 1968.

S. scaber Barbour \& Ramsden 1919-Double-collared Geckolet

S. schuberti Thomas \& Hedges 1998-Neiba Agave Geckolet

S. schwartzi Thomas, Hedges, \& Garrido 1992-Monitongas Collared Geckolet

S. semasiops Thomas 1975-Cockpit Eyespotted Geckolet

S. shrevei Lazell 1961—Northwest Haitian Blotched Geckolet

S. siboney Fong \& Díaz 2004-Siboney Gray-headed Geckolet

S. sommeri Graham 1981—Northwest Haitian Banded Geckolet

S. sputator (Sparrman 1784)—Leeward Banded Geckolet

S. storeyae Grant 1944-Los Canarreos Geckolet

S. streptophorus Thomas \& Schwartz 1977-Hispaniolan Small-eared Geckolet

Subspecies. Sphaerodactylus streptophorus streptophorus Thomas \& Schwartz 1977; S. s. sphenophanes Thomas \& Schwartz 1983.

S. thompsoni Schwartz \& Franz 1976-Barahona Limestone Geckolet

S. torrei Barbour 1914-Cuban Broad-banded Geckolet

Subspecies. Sphaerodactylus torrei torrei Barbour 1914; S. t. spielmani Grant 1958.

S. townsendi Grant 1931—Puerto Rican Sandy Geckolet

S. underwoodi Schwartz 1908-Turks Islands Geckolet

S. vincenti Boulenger 1891-Windward Geckolet

Subspecies. Sphaerodactylus vincenti vincenti Boulenger 1891; S. v. adamas Schwartz 1964; S. v. diamesus Schwartz 1964; S. v. josephinae Schwartz 1964; S. v. monilifer Barbour 1921; S. v. pheristus Schwartz 1964; S. v. psammius Schwartz 1964; S. v. ronaldi Schwartz 1964.

Comment. Sphaerodactylus festus is sometimes considered a subspecies of S. vincenti (e.g., Uetz et al. 2018). We follow Surget-Groba \& Thorpe (2013) in not regconizing S. kirbyi Lazell.

S. williamsi Thomas \& Schwartz 1983-Haitian Striped Geckolet

S. zygaena Schwartz \& Thomas 1977-Tiburon Coastal Geckolet

\section{Family Sphenomorphidae Welch 1982-Forest Skinks}

\section{Scincella Mittleman 1950-Ground Skinks}

S. cherriei (Cope 1893)—Brown Forest Skink

\section{Family Teiidae Gray 1827—Ameivas, Groundlizards, Racerunners, Tegus, Whiptails}

\section{Ameiva Meyer 1795-South American Ameivas}

A. ameiva (Linnaeus 1758)-Neotropical Ameiva

Comment. Some authorities (e.g., Uetz et al. 2018) consider "A. ameiva tobagana" to be a subspecies of A. ameiva, which would render the species polytypic. However, Ugueto \& Harvey (2011) considered A. a. tobagana a synonym of $A$. atrigularis.

A. aquilina Barbour 1930—Antillean Ameiva

Comment. This taxon has sometimes been considered a synonym of A. ameiva tobagana (e.g., Uetz et al. 2018), which Ugueto \& Harvey (2011) considered a synonym of A. atrigularis.

A. atrigularis Garman 1887-Venezuelan Ameiva

Comment. This species was introduced to Barbados from Trinidad, according to Watson (2008). 


\section{A. bifrontata Cope 1862-Khaki Ameiva}

Subspecies. Ameiva bifrontata bifrontata Cope 1862; A. b. insulana Ruthven 1924.

Comment. Harvey et al. (2012) suggested that Ameiva bifrontata represents a species complex.

\section{Aspidoscelis Fitzinger 1843-North American Whiptails}

A. cozumelae (Gadow 1906)—Cozumel Whiptail

\section{Cnemidophorus Wagler 1830-South American Whiptails}

C. arubensis Lidth de Jeude 1887-Aruba Whiptail

C. flavissimus Ugueto, Harvey, \& Rivas 2010—Los Frailes Whiptail

C. lemniscatus (Linnaeus 1758)—Rainbow Whiptail

C. leucopsammus Ugueto \& Harvey 2010-Blanquilla Whiptail

C. murinus (Laurenti 1768)-Curaçao Whiptail

C. nigricolor Peters 1873-Venezuelan Island Whiptail

C. rostralis Ugueto \& Harvey 2010—La Tortuga Whiptail

C. ruatanus Barbour 1928-Central American Whiptail

C. ruthveni Burt 1935-Bonaire Whiptail

C. vanzoi Baskin \& Williams 1966-Maria Islands Whiptail

\section{Holcosus Cope 1862-Bristly-flanked Ameivas}

H. undulatus (Wiegmann 1834)—Rainbow Ameiva

Subspecies. Holcosus undulatus miadis Barbour \& Loveridge 1929.

\section{Kentropyx Spix 1825-Keeled Whiptails}

K. borckiana (Peters 1869)—Guyana Whiptail

Comment. Kentropyx borckiana is a parthenogenetic species that likely arose from hybridization between $K$. calcarata and K. striata (Cole et al. 1995; Reeder et al. 2002).

K. striata (Daudin 1802)—Striped Whiptail

\section{Pholidoscelis Fitzinger 1843-West Indian Groundlizards}

P. alboguttatus (Boulenger 1896)—Mona Groundlizard

Comment. This taxon is sometimes considered a subspecies of P. exsul (e.g., Tucker et al. 2017; Uetz et al. 2018). P. atratus (Garman 1888)—Redonda Groundlizard

P. auberi (Cocteau in Cocteau \& Bibron 1838-1843)—Cuban Groundlizard

Subspecies. Pholidoscelis auberi auberi (Cocteau in Cocteau \& Bibron 1838-1843); P. a. abductus (Schwartz 1970); P. a. atrothorax (Schwartz 1970); P. a. behringensis (Lee \& Schwartz 1985); P. a. bilateralis (McCoy 1970); P. a. cacuminis (Schwartz 1970); P. a. citrus (Schwartz 1970); P. a. denticolus (Schwartz 1970); P. a. extorris (Schwartz 1970); P. a. extrarius (Schwartz 1970); P. a. felis (McCoy 1970); P. a. focalis (McCoy 1970); P. a. galbiceps (Schwartz 1970); P. a. garridoi (Schwartz 1970); P. a. gemmeus (Schwartz 1970); P. a. granti (Schwartz 1970); P. a. hardyi (Schwartz 1970); P. a. kingi (McCoy 1970); P. a. Ilanensis (Schwartz 1970); P. a. marcidus (Schwartz 1970); P. a. multilineatus (McCoy 1970); P. a. nigriventris (Gali \& Garrido 1987); P. a. obsoletus (McCoy 1970); P. a. orlandoi (Schwartz \& McCoy 1975); P. a. parvinsulae (Lee \& Schwartz 1985); P. a. paulsoni (Schwartz 1970); P. a. peradustus (Schwartz 1970); P. a. procer (Schwartz 1970); P. a. pullatus (Schwartz 1970); P. a. richmondi (McCoy 1970); P. a. sabulicolor (Schwartz 1970); P. a. sanfelipensis (Garrido 1975); P. schwartzi (Gali \& Garrido 1987); P. a. secta (Schwartz 1970); P. a. sideroxylon (Lee \& Schwartz 1985); P. a. sublestus (Schwartz 1970); P. a. thoracicus (Cope 1863); P. a. ustulatus (Schwartz 1970); P. a. vulturnus (Lee \& Schwartz 1985); and P. a. zugi (Schwartz 1970).

Comments. Harvey et al. (2012) suggested that Pholidoscelis auberi represents a species complex. Pholidoscelis auberi secta was mistakenly emended by Tucker et al. (2017) to P. a. sectus, but the Latin secta (= way or path) is a noun in apposition and should retain its feminine gender. 
P. chrysolaemus (Cope 1868)—Hispaniolan Giant Groundlizard

Subspecies. Pholidoscelis chrysolaemus chrysolaemus (Cope 1868); P. c. abbotti (Noble 1923); P. c. alacris (Schwartz \& Klinikowski 1966); P. c. boekeri (Mertens 1938); P. c. defensor (Schwartz \& Klinikowski 1966); P. c. evulsus (Schwartz \& Klinikowski 1966); P. c. fictus (Schwartz \& Klinikowski 1966); P. c. jacto (Schwartz \& Klinikowski 1966); P. c. parvoris (Schwartz \& Klinikowski 1966); P. c. procax (Schwartz \& Klinikowski 1966); P. c. quadrijugis (Schwartz 1968); P. c. regularis (Fischer 1888); P. c. richardthomasi (Schwartz \& Klinikowski 1966); P. c. secessus (Schwartz \& Klinikowski 1966); P. c. umbratilis (Schwartz \& Klinikowski 1966); P. c. woodi (Cochran 1934).

Comments. Harvey et al. (2012) suggested that Pholidoscelis chrysolaemus represents a species complex. Pholidoscelis chrysolaemus evulsus is an emendation to reconcile gender with the masculine generic name (fide Tucker et al. 2017).

P. cineraceus (Barbour and Noble 1915)—Guadeloupean Groundlizard

Comments. This species presumably is extinct (Dewynter 2017). Bochaton et al. (2017) suggested that P. major and $P$. cineraceus might be conspecific.

P. corax (Censky \& Paulson 1992)—Little Scrub Groundlizard

P. corvinus (Cope 1861) - Sombrero Groundlizard

P. desechensis (Heatwole \& Torres 1967)—Desecheo Groundlizard

Comment. This taxon is sometimes considered a subspecies of P. exsul (e.g., Tucker et al. 2017; Uetz et al. 2018).

P. dorsalis (Gray 1838)—Jamaican Groundlizard

P. erythrocephalus (Daudin 1802)—Red-faced Groundlizard

P. exsul (Cope 1862)—Puerto Rican Giant Groundlizard

Comment. Pholidoscelis alboguttatus and P. desechensis are sometimes considered subspecies of $P$. exsul (e.g., Tucker et al. 2017; Uetz et al. 2018).

P. fuscatus (Garman 1887)—Dominica Groundlizard

P. griswoldi (Barbour 1916)—Antigua Bank Groundlizard

P. lineolatus (Duméril \& Bibron 1839)—Pygmy Blue-tailed Groundlizard

Subspecies. Pholidoscelis lineolatus lineolatus (Duméril \& Bibron 1839); P. I. beatensis (Noble 1923); P. I. meracuIus (Schwartz 1965); P. I. perplicatus (Schwartz 1965); P. I. privigna (Schwartz 1965); P. I. semotus (Schwartz 1965).

P. major (Duméril \& Bibron 1839)—Guadeloupean Giant Groundlizard

Comments. This species presumably is extinct (but see Dewynter \& Powell 2017). Bochaton et al. (2017) suggested that $P$. major and $P$. cineraceus might be conspecific.

P. maynardii (Garman 1888) —Inagua Groundlizard

Subspecies. Pholidoscelis maynardii maynardii (Garman 1888); P. m. parvinaguae (Barbour \& Shreve 1936); $P$. m. uniformis (Noble \& Klingel 1932).

P. plei (Duméril \& Bibron 1839)—Anguilla Bank Groundlizard

Subspecies. Pholidoscelis plei plei (Duméril \& Bibron 1839); P. p. analiferus (Censky \& Paulson 1992).

P. pluvianotatus (Garman 1887)—Montserrat Groundlizard

P. polops (Cope 1862)—Saint Croix Groundlizard

P. taeniurus (Cope 1862)—Hispaniolan Blue-tailed Groundlizard

Subspecies. Pholidoscelis taeniurus taeniurus (Cope 1862); P. t. aequoreus (Schwartz 1967); P. t. azuae (Schwartz 1967); P. t. barbouri (Cochran 1928); P. t. ignobilis (Schwartz 1967); P. t. meyerabichi (Mertens 1950); P. t. navassae (Schmidt 1919); P. t. pentamerinthus (Schwartz 1968); P. t. regnatrix (Schwartz 1967); P. t. rosamondae (Cochran 1934); P. t. tofaceus (Schwartz 1967); P. t. vafer (Schwartz 1967); P. t. varicus (Schwartz 1967); P. t. vulcanalis (Schwartz 1967).

Comments. Pholidoscelis taeniurus navassae is likely based on a mislabeled specimen from the Tiburon Peninsula of Haiti (Thomas 1966; Powell 1999); if true, Schwartz (1967) indicated that P. t. regnatrix would take the name P. t. navassae. Pholidoscelis t. tofaceus is an emendation to reconcile gender with the masculine generic 
name (fide Tucker et al. 2017).

P. turukaeraensis Bochaton, Boistel, Grouard, Ineich, Tresset, \& Bailon 2017-Marie-Galante Groundlizard

Comment. This taxon is known only from fossil material (Bochaton et al. 2017).

P. wetmorei (Stejneger 1913)—Puerto Rican Blue-tailed Groundlizard

\section{Tupinambis Daudin 1802-Tegus}

T. cryptus Murphy, Jowers, Lehtinen, Charles, Colli, Peres Jr., Hendry, \& Pyron 2016—Cryptic Golden Tegu

Comment. Populations of this species were until recently assigned to Tupinambis teguixin (Muphy et al. 2016).

\section{Family Tropiduridae Bell 1843_Lava, Whorl-tailed, \& Thorny-tailed Lizards, Treerunners}

\section{Plica Gray 1831-Treerunners}

P. caribeana Murphy \& Jowers 2013-Caribbean Treerunner

P. plica (Linnaeus 1758)—Collared Treerunner

Comment. Presence in the region is based on a single specimen presumably taken on Grenada (Henderson \& Murphy 2012; Henderson \& Powell 2018).

\section{Tropidurus Wied-Neuwied 1824-Lava Lizards}

T. hispidus (Spix 1825)—Neotropical Lava Lizard

\section{Family Xantusiidae Baird 1858-Night Lizards}

\section{Cricosaura-Cuban Night Lizards}

C. typica Gundlach \& Peters in Peters 1863-Cuban Night Lizard

$$
\text { Squamata Oppel } 1811 \text { (part)-Snakes }
$$

Family Anomalepididae H. M. Smith 1966-Divergent Blindsnakes

\section{Liotyphlops Peters 1881-Wide-nosed Blindsnakes}

L. albirostris (Peters 1857)—White-nosed Blindsnake

\section{Family Boidae Gray 1825-Boas, Anacondas}

\section{Boa Linnaeus 1758-Typical Boas}

B. blanchardensis Bochaton \& Bailon 2018-Marie-Galante Boa

Comment. This species is known only from fossil material (Bochaton \& Bailon 2018).

B. constrictor Linnaeus 1758-Boa constrictor

Subspecies. Boa constrictor constrictor Linnaeus 1758

B. imperator Daudin 1803-Central American Boa

Subspecies. Boa imperator imperator Daudin 1803

B. nebulosa (Lazell 1964)—Dominica Boa

B. orophias Linnaeus 1758-Saint Lucia Boa

\section{Chilabothrus Duméril \& Bibron 1844-West Indian Boas}

C. angulifer (Bibron 1843)—Cuban Boa

C. argentum Reynolds, Puente-Rolón, Geneva, Avilés-Rodríguez, \& Herrmann 2016-Conception Bank Boa

C. chrysogaster (Cope 1871)-Turks \& Caicos Boa

Subspecies. Chilabothrus chrysogaster chrysogaster (Cope 1871); C. c. relicquus (Barbour \& Shreve 1935).

C. exsul (Netting \& Goin 1944)_Abaco Islands Boa 
C. fordii (Günther 1861)—Hispaniolan Desert Boa

Subspecies. Chilabotrus fordii fordii (Günther 1861); C. f. agametus (Sheplan \& Schwartz 1974); C. f. manototus (Schwartz 1979).

C. gracilis Fischer 1888-Hispaniolan Gracile Boa

Subspecies. Chilabothrus gracilis gracilis Fischer 1888; C. g. hapalus (Sheplan \& Schwartz 1974).

C. granti (Stull 1933) -Virgin Islands Boa

C. inornatus (Reinhardt 1843)_Puerto Rican Boa

C. monensis (Zenneck 1898) - Mona Boa

C. schwartzi (Buden 1975)—Crooked-Aklins Boa

C. striatus (Fischer 1856)—Hispaniolan Boa

Subspecies. Chilabothrus striatus striatus (Fischer 1856); C. s. exagistus (Sheplan \& Schwartz 1974); C. s. warreni (Sheplan \& Schwartz 1974).

C. strigilatus (Cope 1863)—Bahamian Boa

Subspecies. Chilabothrus strigilatus strigilatus (Cope 1863); C. s. ailurus (Sheplan \& Schwartz 1974); C. s. fosteri (Barbour 1941); C. s. fowleri (Sheplan \& Schwartz 1974).

C. subflavus (Stejneger 1901)—Jamaican Boa

\section{Corallus Daudin 1803-Treeboas}

C. cookii Gray 1842-Saint Vincent Treeboa

Comment. Colston et al. (2013) found a close relationship between this species and C. hortulanus, noting that additional research is needed to determine the taxonomic status of $C$. cookii.

C. grenadensis (Barbour 1914)—Grenada Bank Treeboa

Comment. Colston et al. (2013) found a close relationship between this species and C. hortulanus, noting that additional research is needed to determine the taxonomic status of $C$. grenadensis.

C. ruschenbergerii (Cope 1875)—Mangrove Treeboa

\section{Epicrates Wagler 1830-Rainbow Boas}

E. maurus Gray 1849-Brown Rainbow Boa

\section{Eunectes Wagler 1830-Anacondas}

E. murinus Linnaeus 1758-Green Anaconda

\section{Family Colubridae Oppel 1811—Common Snakes}

\section{Chironius Fitzinger 1826-Neotropical Coachwhips}

C. carinatus (Linnaeus 1758)-Amazon Coachwhip

C. septentrionalis Dixon, West, \& Cei 1993-South American Coachwhip

C. vincenti (Boulenger 1891)—Saint Vincent Coachwhip

\section{Drymarchon Fitzinger 1843-Indigo Snakes}

D. corais (Boie 1827)-Western Indigo Snake

D. melanurus (Duméril, Bibron, \& Duméril 1854)—Central American Indigo Snake

Subspecies. Drymarchon melanurus melanurus (Duméril, Bibron, \& Duméril 1854).

\section{Drymobius Fitzinger 1843-Neotropical Racers}

D. margaritiferus (Schlegel 1837)—Northern Speckled Racer

Subspecies. Drymobius margaritiferus margaritiferus (Schlegel 1837) D. m. maydis Villa 1968.

\section{Leptophis Bell 1825-Parrot Snakes}

L. coeruleodorsus Oliver 1942-Green-and-yellow Parrot Snake 
L. haileyi Murphy Charles Lehtinen \& Koeller 2013-Tobagan Parrot Snake

L. mexicanus Duméril, Bibron, \& Duméril 1854-Mexican Parrot Snake

Subspecies. Leptophis mexicanus mexicanus Duméril Bibron \& Duméril 1854; L. m. hoeversi Henderson 1976.

L. stimsoni Harding 1995-Trinidad Upland Parrot Snake

\section{Mastigodryas Amaral 1935-Treeracers}

M. boddaerti (Sentzen 1796)—Common Treeracer

Subspecies. Mastigodryas boddaerti dunni (Stuart 1933)

M. bruesi (Barbour 1914)—Windward Treeracer

M. melanolomus (Cope 1868)-Salmon-bellied Treeracer

Subspecies. Mastigodryas melanolomus melanolomus (Cope 1868).

M. pleei (Duméril, Bibron, \& Duméril) 1854-Blotched Treeracer

\section{Oxybelis Wagler 1830-Neotropical Vinesnakes}

O. aeneus (Wagler 1824)—Brown Vinesnake

O. fulgidus (Daudin 1803)—Green Vinesnake

O. wilsoni Villa \& McCranie 1995-Roatan Vinesnake

\section{Pantherophis Fitzinger 1843-North American Ratsnakes}

P. guttatus (Linnaeus 1766)—Red Cornsnake

\section{Phrynonax Cope 1862-Puffing Snakes}

P. polylepis (Peters 1867)—Northeastern Puffing Snake

\section{Pseudelaphe Mertens \& Rosenberg 1943-Middle American Ratsnakes}

P. flavirufa (Cope 1867)-Yellow-red Ratsnake

Subspecies. Pseudelaphe flavirufa pardalina (Peters 1868).

\section{Pseudoboa Schneider 1801-Falseboas}

P. neuwiedii (Duméril, Bibron, \& Duméril 1854)—Dark-headed Red Falseboa

\section{Spilotes Wagler 1830—Chicken Snakes, Hissing Snakes}

S. pullatus (Linnaeus 1758)-Tropical Chicken Snake

S. sulphureus (Wagler 1824)-Yellow-bellied Hissing Snake

\section{Tantilla Baird \& Girard 1853-Black-headed Snakes, Centipede Snakes}

T. melanocephala (Linnaeus 1758)—Neotropical Black-headed Snake

T. tritaeniata Smith \& Williams 1966-Three-banded Centipede Snake

\section{Tantillita Smith 1941—Dwarf Short-tailed Snakes}

T. lintoni (Smith 1940)—Brown Dwarf Short-tailed Snake

\section{Family Dipsadidae Bonaparte 1838-Neotropical Rear-fanged Snakes}

\section{Alsophis Fitzinger 1843-Lesser Antillean Racers}

A. antiguae Parker 1933-Antiguan Racer

A. antillensis (Schlegel 1837)—Guadeloupe Racer

A. manselli Parker 1933-Montserrat Racer

A. rijgersmaei Cope 1869-Anguilla Bank Racer

A. rufiventris (Duméril, Bibron, \& Duméril 1854)—Red-bellied Racer

A. sanctonum Barbour 1915-Terre-De-Haut Racer 
A. sibonius Cope 1879-Dominica Racer

\section{Arrhyton Günther 1858-Racerlets}

A. ainictum Schwartz \& Garrido 1981-Las Tunas Racerlet

A. dolichura Werner 1909-Havana Racerlet

A. procerum Hedges \& Garrido 1992-Zapata Long-tailed Racerlet

A. redimitum (Cope 1862)-Oriente Brown-capped Racerlet

A. supernum Hedges \& Garrido 1992-Oriente Black Racerlet

A. taeniatum Günther 1858-Broad-striped Racerlet

A. tanyplectum Schwartz \& Garrido 1981—Guaniguanico Racerlet

A. vittatum (Gundlach in Peters 1861)—Cuban Short-tailed Racerlet

\section{Atractus Wagler 1828-Neotropical Groundsnakes}

A. trilineatus Wagler 1828-Three-lined Groundsnake

A. univittatus Jan 1862-One-lined Groundsnake

\section{Borikenophis Hedges \& Vidal 2009-Puerto Rican Racers}

B. portoricensis (Reinhardt \& Lütken 1862)—Puerto Rican Racer

Subspecies. Borikenophis portoricensis portoricensis (Reinhardt \& Lütken 1862); B. p. anegadae (Barbour 1917);

B. p. aphantus (Schwartz 1966); B. p. nicholsi (Grant 1937); B. p. prymnus (Schwartz 1966); B. p. richardi (Grant 1946).

B. sanctaecrucis (Cope 1862)—Saint Croix Racer

Comment. This species almost certainly is extinct (e.g., Henderson \& Powell 1996; Platenberg \& Powell 2016).

B. variegatus (Schmidt 1926)—Mona Racer

\section{Caraiba Zaher, Grazziotin, Cadle, Murphy, Moura-Leite, \& Boanatto 2009-Cuban Lesser Racers}

C. andreae (Reinhardt \& Lütken 1862)—Cuban Lesser Racer

\section{Clelia Fitzinger 1826-Cribos}

C. clelia (Daudin 1803)—Windward Cribo

Comment. This species probably is extirpated on Grenada (e.g., Henderson \& Powell 2018).

C. errabunda Underwood 1993-Saint Lucia Cribo

Comment. This species is almost certainly extinct (e.g., Daltry 2009; Daltry et al. 2016).

\section{Coniophanes Hallowell 1860—Smooth-scaled Groundsnakes}

C. andresensis Bailey 1937-San Andres Snake

C. bipunctatus (Günther 1858)-Two-spotted Snake

C. imperialis (Baird \& Girard 1859)—Black-striped Snake

Subspecies. Coniophanes imperialis clavatus (Peters 1864)

\section{Cubophis Hedges and Vidal 2009-North Caribbean Racers}

C. brooksi (Barbour 1914)—Swan Island Racer

C. cantherigerus (Bibron 1843)—Cuban Racer

Subspecies. Cubophis cantherigerus cantherigerus (Bibron 1843); C. c. adspersus (Gundlach \& Peters 1864); C. C. pepei (Schwartz \& Thomas 1960); C. c. schwartzi (Lando \& Williams 1969).

C. caymanus (Garman 1887)—Grand Cayman Racer

C. fuscicauda (Garman 1888)—Cayman Brac Racer

C. ruttyi (Grant 1941)—Little Cayman Racer

C. vudii (Cope 1862)-Bahamian Racer

Subspecies. Cubophis vudii vudii (Cope 1862); C. v. aterrimus (Barbour \& Shreve 1935); C. v. picticeps (Conant 
1937); C. v. raineyi (Barbour \& Shreve 1935); C. v. utowanae (Barbour \& Shreve 1935).

\section{Dipsas Laurenti 1768-Snail-eaters}

D. trinitatis Parker 1926-Trinidad Snail-eater

D. variegata (Duméril, Bibron, \& Duméril 1854)—Variegated Snail-eater

Subspecies. Dipsas variegata nicholsi (Dunn 1933).

\section{Enulius Cope 1871-American Long-tailed Snakes}

E. bifoveatus McCranie \& Köhler 1999—Guanaja Long-tailed Snake

E. roatanensis McCranie \& Köhler 1999—Roatan Long-tailed Snake

\section{Erythrolamprus Wagler 1830-South American False Coralsnakes, Antillean Groundsnakes}

E. aesculapii (Linnaeus 1766)—South American False Coralsnake

E. cobella Lacépède 1789-Mangrove Groundsnake

E. cursor Lacépède 1789-Martinique Groundsnake

Comment. This species might be extinct (Dewynter et al. 2016).

E. juliae (Cope 1879)—Leeward Groundsnake

Subspecies. Erythrolamprus juliae juliae (Cope 1879); E. j. copeae (Parker 1936); E. j. mariae (Barbour 1914).

E. melanotus (Shaw 1802)—Dark Groundsnake

E. ocellatus Peters 1869-Tobago False Coralsnake

E. ornatus (Garman 1887)—Saint Lucia Groundsnake

E. perfuscus (Cope 1862)—Barbados Groundsnake

E. triscalis (Linnaeus 1758) - Three-scaled Groundsnake

E. zweifeli (Roze 1959)—Braided Groundsnake

\section{Haitiophis (Hedges \& Vidal 2009)—Hispaniolan Brown Racers}

H. anomalus (Peters 1863) — Hispaniolan Brown Racer

\section{Helicops Wagler 1830-American Keelbacks}

H. angulatus (Linnaeus 1758)—Brown-banded Watersnake

\section{Hydrops Wagler 1830-South American Watersnakes}

H. triangularis Wagler 1824-Triangle Watersnake

\section{Hypsirhynchus Günther 1858-Antillean Groundracers}

H. ater (Gosse 1851)—Jamaican Giant Racer

Comment. This species might be extinct (Hedges et al. 2016).

H. callilaemus (Gosse 1851)—Jamaican Red Racer

H. ferox Günther 1858-Hispaniolan Hog-nosed Racer

H. funereus (Cope 1863)—Jamaican Black Racer

H. melanichnus (Cope 1862)—Hispaniolan Olive Racer

Comment. This species might be extinct (Landestoy et al. 2016).

H. parvifrons (Cope 1862)-Common Hispaniolan Racer

Subspecies. Hypsirhynchus parvifrons parvifrons (Cope 1862); H. p. alleni (Dunn 1920); H. p. lincolni (Cochran 1941); H. p. niger (Dunn 1920); H. p. paraniger (Thomas \& Schwartz 1965); H. p. protenus (Jan 1867); H. p. rosamondae (Cochran 1934); H. p. stygius (Thomas \& Schwartz 1965); H. p. tortuganus (Dunn 1920).

H. polylepis (Buden 1966)—Jamaican Long-tailed Racer

H. scalaris Cope 1863-Tiburon Hog-nosed Racer

\section{Ialtris Cope 1862-Hispaniolan Forest Racers}

I. agyrtes Schwartz \& Rossman 1976—Barahona Red-headed Racer 
I. dorsalis (Günther 1858)—Hispaniolan W-headed Racer

I. haetianus (Cochran 1935)—Hispaniolan Upland Racer

Subspecies. Ialtris haetianus haetianus (Cochran 1935); I. h. perfector (Schwartz \& Thomas 1965); I. h. vaticinata (Schwartz 1970).

I. parishi Cochran 1932-Tiburon Banded Racer

Imantodes Duméril 1853-Blunt-headed Treesnakes

I. cenchoa (Linnaeus 1758)—Neotropical Blunt-headed Treesnake

Leptodeira Fitzinger 1843-Cat-eyed Snakes

L. annulata Linnaeus 1758-Banded Cat-eyed Snake

Subspecies. Leptodeira annulata ashmeadi (Hallowell 1845).

L. bakeri Ruthven 1936-Aruban Cat-eyed Snake

L. frenata Cope 1886-Mayan Cat-eyed Snake

Subspecies. Leptodeira frenata malleisi Dunn \& Stuart 1935.

Magliophis Zaher, Grazziotin, Cadle, Murphy, Moura-Leite, \& Bonatto 2009-Miniracers

M. exiguus Cope 1862-Virgin Islands Miniracer

Subspecies. Magliophis exiguus exiguus (Cope 1862); M. e. subspadix Schwartz 1967.

M. stahli Stejneger 1904-Puerto Rican Miniracer

Ninia Baird \& Girard 1853-Coffeesnakes

N. atrata (Hallowell 1845)-South American Coffeesnake

N. francisco Angarita-Sierra 2014-Simla Coffeesnake

Comment. This species is known only from the holotype (Angarita-Sierra 2014).

\section{Oxyrhopus Wagler 1830-Neotropical False Coralsnakes}

O. petolarius (Linnaeus 1758)-Forest Flamesnake

Subspecies. Oxyrhopus petolarius petolarius (Linnaeus 1758).

\section{Sibon Fitzinger 1826-Snail-eating Snakes}

S. nebulatus (Linnaeus 1758)—Cloudy Snail-eating Snake

Subspecies. Sibon nebulata nebulata (Linnaeus 1758).

\section{Siphlophis Fitziger 1843-Neotropical Nightsnakes}

S. cervinus (Laurenti 1768)—Panamanian Spotted Nightsnake

S. compressus (Daudin 1803)-Tropical Flatsnake

\section{Thamnodynastes Wagler 1830-American House Snakes}

T. ramonriveroi Manzanilla \& Sánchez 2005-Guianan Coastal House Snake

\section{Tretanorhinus Duméril, Bibron, \& Duméril 1854-Neotropical Swampsnakes}

T. nigroluteus Cope 1861—Orange-bellied Swampsnake

T. variabilis Duméril, Bibron, \& Duméril 1854-Caribbean Watersnake

Subspecies. Tretanorhinus variabilis variabilis Duméril, Bibron, \& Duméril 1854; T. v. binghami Schwartz \& Ogren 1956; T. v. insulaepinorum Barbour 1916; T. v. lewisi Grant 1941; T. v. wagleri Jan 1863.

\section{Uromacer Duméril \& Bibron 1853-Hispaniolan Vinesnakes}

U. catesbyi (Schlegel 1837)—Blunt-headed Hispaniolan Vinesnake

Subspecies. Uromacer catesbyi catesbyi (Schlegel 1837); U. c. cereolineatus Schwartz 1970; U. c. frondicolor Schwartz 1970; U.c. hariolatus Schwartz 1970; U. c. inchausteguii Schwartz 1970; U. c. insulaevaccarum Schwartz 1970; U. c. pampineus Schwartz 1970; U. c. scandax Dunn 1920. 
U. frenatus (Günther 1865)—Slender Hispaniolan Vinesnake

Subspecies. Uromacer frenatus frenatus (Günther 1865); U. f. chlorauges Schwartz 1976; U. f. dorsalis Dunn 1920; U. f. wetmorei Cochran 1931.

U. oxyrhynchus Duméril, Bibron, \& Duméril 1854-Sharp-nosed Hispaniolan Vinesnake

\section{Family Elapidae F. Boie 1827-Front-fanged Snakes}

\section{Micrurus Wagler 1824-American Coralsnakes}

M. circinalis (Duméril, Bibron, \& Duméril 1854)—Trinidadian Coralsnake

M. lemniscatus (Linnaeus 1758)—South American Small-eyed Coralsnake

Subspecies. Micrurus lemniscatus diutius (Burger 1955).

M. nigrocinctus Girard 1854-Central American Coralsnake

Subspecies. Micrurus nigrocinctus babaspul (Roze 1967).

M. ruatanus (Günther 1895)—Roatan Coralsnake

\section{Family Leptotyphlopidae Stejneger 1892-Threadsnakes}

\section{Epictia Gray 1845-Neotropical Threadsnakes}

E. columbi (Klauber 1939)—Bahamian Threadsnake

E. goudotii (Duméril \& Bibron 1844)—Southern Caribbean Threadsnake

E. magnamaculata (Taylor 1940)—Western Caribbean Threadsnake

E. tenella (Klauber 1939)—Guyana Threadsnake

\section{Mitophis Hedges, Adalsteinsson, \& Branch 2009-Hispaniolan Threadsnakes}

M. asbolepis (Thomas, McDiarmid, \& Thompson 1985)—Martin Garcia Threadsnake

M. calypso (Thomas, McDiarmid, \& Thompson 1985)—Samana Threadsnake

M. leptepileptus (Thomas, McDiarmid, \& Thompson 1985)—La Selle Threadsnake

M. pyrites (Thomas 1965)—Barahona Threadsnake

\section{Tetracheilostoma Jan 1861-Lesser Antillean Threadsnakes}

T. bilineatum Schlegel 1839-Martinique Threadsnake

T. breuili Hedges 2008-Saint Lucia Threadsnake

T. carlae Hedges 2008-Barbados Threadsnake

\section{Family Natricidae Bonaparte 1838-Northern Hemisphere Keeled Snakes}

\section{Nerodia Baird \& Girard 1853-North American Watersnakes}

N. clarkii (Baird \& Girard 1853)—Saltmarsh Snake

Subspecies. Nerodia clarkii compressicauda Kennicott 1860.

\section{Thamnophis Fitzinger 1843-Gartersnakes and Ribbonsnakes}

T. proximus (Say 1823) —Western Ribbonsnake

Subspecies. Thamnophis proximus rutiloris (Cope 1885).

\section{Tropidophiidae Brongersma 1951—Tropes, Eyelash Boas}

\section{Tropidophis Bibron 1840-Tropes}

T. bucculentus Cope 1868-Navassa Trope

T. canus Cope 1868-Inagua Trope 
T. caymanensis Battersby 1938-Grand Cayman Trope

T. celiae Hedges, Estrada, \& Díaz 1999-Canasi Trope

T. curtus Garman 1887-Northern Bahamas Trope

T. feicki Schwartz 1957-Broad-banded Trope

T. fuscus Hedges \& Garrido 1992—Cuban Dusky Trope

T. galacelidus Schwartz \& Garrido 1975-Escambray White-necked Trope

T. greenwayi Barbour \& Shreve 1936-Caicos Trope

Subspecies. Tropidophis greenwayi greenwayi Barbour \& Shreve 1936; T. g. Ianthanus Schwartz 1963.

T. haetianus Cope 1879-Hispaniolan Trope

Subspecies. Tropidophis haetianus haetianus Cope 1879; T. h. hemerus Schwartz 1975; T. h. tiburonensis Schwartz 1975.

T. hardyi Schwartz \& Garrido 1975-Escambray Small-headed Trope

T. hendersoni Hedges \& Garrido 2002-Cuban Khaki Trope

T. jamaicensis Stull 1928-Jamaican Brown Trope

T. maculatus Bibron 1843-Spotted Red Trope

T. melanurus Schlegel 1837-Giant Trope

Subspecies. Tropidophis melanurus melanurus (Schlegel 1837); T. m. dysodes Schwartz \& Thomas 1960; T. m. ericksoni Schwartz \& Thomas 1960.

T. morenoi Hedges \& Garrido 2001-Zebra Trope

T. nigriventris Bailey 1937—Dark-bellied Trope

T. pardalis Gundlach 1840—Spotted Brown Trope

T. parkeri Grant 1941—Little Cayman Trope

T. pilsbryi Bailey 1937-Oriente White-necked Trope

T. schwartzi Thomas 1963-Cayman Brac Trope

T. semicinctus Gundlach \& Peters in Peters 1865-Yellow-banded Trope

T. spiritus Hedges \& Garrido 1999—Sancti Spiritus Trope

T. stejnegeri Grant 1940—Jamaican Eyespotted Trope

T. stullae Grant 1940—Portland Ridge Trope

T. wrighti Stull 1938-Gracile Banded Trope

T. xanthogaster Domínguez, Moreno, \& Hedges 2006-Guanahacabibes Trope

\section{Family Typhlopidae Merrem 1820-Typical Blindsnakes}

\section{Amerotyphlops Hedges, Marion, Lipp, Marin, \& Vidal 2014-American Blindsnakes}

A. brongersmianus (Vanzolini 1976)—South American Striped Blindsnake

A. tasymicris (Thomas 1974)—Grenada Bank Blindsnake

A. trinitatus (Richmond 1965) - Trinidad Blindsnake

\section{Antillotyphlops Hedges, Marion, Lipp, Marin, \& Vidal 2014-East Caribbean Blindsnakes}

A. annae (Breuil 1999)—Saint Barts Blindsnake

A. catapontus (Thomas 1966) - Anegada Blindsnake

A. dominicanus (Stejneger 1904)—Dominica Blindsnake

A. geotomus (Thomas 1966)—Leeward Blindsnake

A. granti (Ruthven \& Gaige 1935)—Guanica Blindsnake

A. guadeloupensis (Richmond 1966)—Guadeloupe Blindsnake

A. hypomethes (Hedges \& Thomas 1991)—Puerto Rican Coastal Blindsnake

A. monastus (Thomas 1966)—Montserrat Blindsnake 
A. monensis (Schmidt 1926)—Mona Blindsnake

A. naugus (Thomas 1965) - Virgin Gorda Blindsnake

A. platycephalus (Duméril \& Bibron 1844)—Puerto Rican White-tailed Blindsnake

A. richardii (Duméril \& Bibron 1844)—Virgin Islands Blindsnake

\section{Cubatyphlops Hedges, Marion, Lipp, Marin, \& Vidal 2014-Cuban Blindsnakes}

C. anchaurus (Thomas \& Hedges 2007)—Maisi Blindsnake

C. anousius (Thomas \& Hedges 2007)—Cuban Pallid Blindsnake

C. arator (Thomas \& Hedges 2007)—Havana Giant Blindsnake

C. biminiensis (Richmond 1955)—Bahaman Slender Blindsnake

C. caymanensis (Sackett 1940)—Grand Cayman Blindsnake

C. contorhinus (Thomas \& Hedges 2007)—Cuban Short-nosed Blindsnake

C. epactius (Thomas 1968)—Cayman Brac Blindsnake

C. golyathi (Domínguez \& Moreno 2009)—Pinar Del Rio Giant Blindsnake

C. notorachius (Thomas \& Hedges 2007)—Imias Blindsnake

C. paradoxus (Thomas 1968)—Inagua Blindsnake

C. perimychus (Thomas \& Hedges 2007)—Guantanamo Bay Blindsnake

C. satelles (Thomas \& Hedges 2007)—Cienfuegos Blindsnake

\section{Indotyphlops Hedges, Marion, Lipp, Marin, \& Vidal 2014-South Asian Blindsnakes}

I. braminus (Daudin 1803)—Brahminy Blindsnake

\section{Typhlops Oppel 1811-West Caribbean Blindsnakes}

T. agoralionis Thomas \& Hedges 2007—La Hotte Blindsnake

T. capitulatus Richmond 1964-Haitian Pale-lipped Blindsnake

T. eperopeus Thomas \& Hedges 2007-Baoruco Blindsnake

T. gonavensis Richmond 1964-Gonave Blindsnake

T. hectus Thomas 1974-Tiburon Peninsula Blindsnake

T. jamaicensis Shaw 1802-Jamaican Blindsnake

T. lumbricalis Linnaeus 1758-Cuban Brown Blindsnake

Comment. We treat Typhlops oxyrhinus Dominguez \& Diaz 2011, Typhlops pachyrhinus Dominguez \& Diaz 2011, and Typhlops leptolepis Dominguez, Fong, \& Iturriaga 2013 as synonymns of Typhlops lumbricalis because an insufficient sampling of museum material was used to diagnose them. A comprehensive molecular and morphological review of Typhlops lumbricalis (R. Thomas and S. B. Hedges, unpublished) does not support the regconition of those taxa as described.

T. proancylops Thomas \& Hedges 2007-La Selle Blindsnake

T. pusillus Barbour 1914-Hispaniolan Common Blindsnake

T. rostellatus Stejneger 1904-Puerto Rican Brown-bellied Blindsnake

T. schwartzi Thomas 1989-Hispaniolan Giant Blindsnake

T. silus Legler 1959-Oriente Blindsnake

T. sulcatus Cope 1868-Hispaniolan Bicolored Blindsnake

T. sylleptor Thomas \& Hedges 2007-Pestel Blindsnake

T. syntherus Thomas 1965-Barahona Peninsula Blindsnake

T. tetrathyreus Thomas 1989-Port-Au-Prince Blindsnake

T. titanops Thomas 1989—Big-eyed Blindsnake

\section{Family Viperidae Oppel 1811-Vipers}




\section{Bothrops Wagler 1824-Lanceheads}

B. atrox (Linnaeus 1758)-Common Lancehead

B. caribbaeus (Garman 1887)-Saint Lucia Lancehead

B. lanceolatus (Lacépède 1789)—Martinique Lancehead

Comment. The name "Fer-de-lance" frequently is used indiscriminately for all Lanceheads, but was originally coined by Lacépède (1789) for B. lanceolatus and is accurately used solely for that species (Minton \& Minton 1969; Cendrero et al. 1972).

\section{Crotalus Linnaeus 1758-Typical Rattlesnakes}

C. durissus Linnaeus 1758-Cascabel Rattlesnake

Subspecies. Crotalus durissus cumanensis Humboldt 1811; C. d. unicolor Lidth de Jeude 1887.

\section{Lachesis Daudin 1803-Bushmasters}

L. muta (Linnaeus 1766)—South American Bushmaster

\section{Testudines Batsch 1788-Turtles \\ Family Cheloniidae Oppel 1811-Seaturtles}

\section{Caretta Rafinesque 1814-Loggerhead Seaturtles}

C. caretta (Linnaeus 1758)—Loggerhead Seaturtle

\section{Chelonia Brongniart 1800-Green Seaturtles}

C. mydas (Linnaeus 1758)—Green Seaturtle

\section{Eretmochelys Fitzinger 1843-Hawksbill Seaturtles}

E. imbricata (Linnaeus 1766)—Hawksbill Seaturtle

\section{Lepidochelys Fitzinger 1843-Ridley Seaturtles}

L. kempii (Garman 1880)—Kemp's Ridley Seaturtle

L. olivacea (Eschscholtz 1829)_Olive Ridley Seaturtle

\section{Family Dermochelyidae Fitzinger 1843-Leatherback Seaturtles}

\section{Dermochelys Blainville 1816-Leatherback Seaturtles}

D. coriacea (Vandelli 1761)—Leatherback Seaturtle

\section{Family Emydidae Rafinesque 1815—Pond Turtles}

\section{Trachemys Agassiz 1857-Sliders}

T. decorata (Barbour \& Carr 1940)—Haitian Slider

T. decussata (Gray 1831)—Cuban Slider

Subspecies. Trachemys decussata decussata (Gray 1831); T. d. angusta (Barbour \& Carr 1940).

T. ornata (Gray 1830)—Ornate Slider

T. scripta (Thunberg in Schoepff 1792)—Pondslider

Subspecies. Trachemys scripta elegans (Wied 1838).

T. stejnegeri (Schmidt 1928)—Antillean Slider

Subspecies. Trachemys stejnegeri stejnegeri (Schmidt 1928); T. s. vicina (Barbour \& Carr 1940); T. s. malonei (Barbour \& Carr 1938).

T. terrapen (Lacépède 1788)—Jamaican Slider 
Family Geoemydidae Theobald 1868-Neotropical Wood Turtles, Eurasian Pond \& River Turtles

\section{Rhinoclemmys Fitzinger 1835-Neotropical Wood Turtles}

R. areolata (Duméril, Bibron, \& Duméril 1851)—Furrowed Wood Turtle

R. punctularia (Daudin 1801)—Spotted-legged Turtle

Subspecies. Rhinoclemmys punctularia punctularia Daudin 1801).

\section{Family Kinosternidae Agassiz 1857-American Mud \& Musk Turtles}

\section{Kinosternon Spix 1824-American Mud Turtles}

K. leucostomum (Duméril, Bibron, \& Duméril 1851)—White-lipped Mud Turtle

K. scorpioides (Linnaeus 1766)-Scorpion Mud Turtle

Subspecies. Kinosternon scorpioides albogulare (Duméril \& Bocourt 1870).

\section{Family Testudinidae Batsch 1788-Tortoises}

\section{Centrochelys Gray 1872-Spurred tortoises}

C. sulcata (Miller 1779)—African Spurred Tortoise

\section{Chelonoidis Fitzinger 1835-Neotropical Tortoises}

Comment. Tortoises known only from fossil material but not assigned to any species (i.e., identified only as "Chelonoidis sp.") are known from the Turks \& Caicos Islands (presumably two species), Curaçao, Hispaniola, and Navassa, plus "limited fragmentary material," known from Barbados, Anguilla, and the Bahamas (Great Bahama, San Salvador, Crooked-Acklins, and Mayaguana Banks), "needs further analysis and hopefully additional material to better elucidate their relationships" (Turtle Extinctions Working Group 2015).

C. alburyorum (Franz \& Franz 2009)—Abaco Tortoise

Comment. This taxon is known only from fossil material; fragmentary material of an older, larger species preceding C. alburyorum also has been found (Turtle Extinctions Working Group 2015).

C. carbonarius (Spix 1824)—Red-footed Tortoise

C. cubensis (Leidy 1868)—Cuban Giant Tortoise

Comment. This taxon is known only from fossil material (Turtle Extinctions Working Group 2015).

C. denticulatus (Linnaeus 1766)-Yellow-footed Tortoise

C. monensis (Williams 1952)—Mona Tortoise

Comment. This taxon is known only from fossil material (Turtle Extinctions Working Group 2015).

C. sombrerensis (Leidy 1868)—Sombrero Giant Tortoise

Comment. This taxon is known only from fossil material (Turtle Extinctions Working Group 2015).

\section{Crocodylia Owen 1842-Alligators, Crocodiles}

Family Alligatoridae Gray 1844-Alligators, Caimans

\section{Caiman Spix 1825-Caimans}

C. crocodilus (Linnaeus 1758)—Spectacled Caiman

Paleosuchus Gray 1862-Dwarf Caimans

P. palpebrosus (Cuvier 1807)—Dwarf Caiman

Family Crocodylidae Cuvier 1807-Crocodiles

Crocodylus Laurenti 1768-Crocodiles 
C. acutus (Cuvier 1807)—American Crocodile

C. antillensis Varona 1966-Antillean Crocodile

Comments. This species is known only from fossil material (Varona 1966). Citing the original description, Ross (1998) listed this taxon in the synonymy of C. rhombifer, despite Varona (1984) stating that $C$. antillensis was a valid species.

C. rhombifer (Cuvier 1807)—Cuban Crocodile

\section{Acknowledgements}

We thank Angela Lu for assistance in assembling the data and figures, Quyen Le for typesetting, and Graham Reynolds, Roger Thorpe, and an anonymous reviewer for comments on the manuscript.

\section{References}

Acevedo AA, Lampo M, Cipriani R (2016) The cane or marine toad, Rhinella marina (Anura, Bufonidae): Two genetically and morphologically distinct species. Zootaxa, 4103, 574-586.

Alonso R, Crawford AJ, Bermingham E (2012) Molecular phylogeny of an endemic radiation of Cuban toads (Bufonidae: Peltophryne) based on mitochondrial and nuclear genes. Journal of Biogeography 39, 434-451.

Angarita-Sierra T (2014) Hemipenial morphology in the semifossorial snakes of the genus Ninia and a new species from Trinidad, West Indies (Serpentes: Dipsadidae). South American Journal of Herpetology, 9, 114-130.

Barbour T (1914) A contribution to the zoögeography of the West Indies, with especial reference to amphibians and reptiles. Memoirs of the Museum of Comparative Zoölogy at Harvard College, 44, 205-359.

Bochaton, C., Bailon, S. (2018) A new fossil species of Boa Linnaeus, 1758 (Squamata, Boidae), from the Pleistocene of Marie-Galante Island (French West Indies). Journal of Paleontology, DOI: 10.1080/02724634.1462829.

Bochaton C, Boistel R, Cassagrande F, Grouard S, Bailon S (2016) A fossil Diploglossus (Squamata, Anguidae) lizard from Basse-Terre and Grande-Terre islands (Guadeloupe, French West-Indies). Scientific Reports, 28475, $1-12$.

Bochaton C, Boistel R, Grouard S, Ineich I, Tresset A, Bailon S (2017) Evolution, diversity and interactions with past human populations of recently extinct Pholidoscelis lizards (Squamata: Teiidae) from the Guadeloupe Islands (French West-Indies). Historical Biology, DOI: 10.1080/08912963.2017.1343824.

Böhme W (1984) Erstfund eines fossilien Kugelfingergeckos (Sauria: Gekkonidae: Sphaerodactylinae) aus Dominikanischem Bernstein (Oligozän von Hispaniola, Antillen). Salamandra, 20, 212-220.

Bond J (1960) Birds of the West Indies. London: Collins.

Breuil M (2002) Histoire naturelle des amphibiens et reptiles terrestres de l'archipel Guadeloupéen. Guadeloupe, Saint-Martin, Saint Barthélemy. Patrimoines Naturels, Paris, 54, 1-339.

Bryan JJ, Gerber GP, Welch ME, Stephen CL (2007) Re-evaluating the taxonomic status of the Booby Cay Iguana, Cyclura carinata bartschi. Copeia, 2007, 734-739.

CARICOM (2018) CARICOM. Caribbean Community. Available online at https://www.caricom.org/ (accessed 10 June 2018). Georgetown, Guyana: CARICOM.

Castañeda MdR, de Queiroz K (2011) Phylogenetic relationships of the Dactyloa clade of Anolis lizards based on nuclear and mitochondrial DNA sequence data. Molecular Phylogenetics and Evolution, 61, 784-800.

Cole CJ, Dessauer HC, Townsend CR, Arnold MG (1995) Kentropyx borckiana (Squamata:Teiidae): an unisexual lizard of hybrid origin in the Guyana region, South America. American Museum Novitates, 2671, 1-5.

Colston TJ, Grazziotin FG, Shepard DB, Vitt CJ, Colli GR, Henderson RW, Hedges SB, Bonatto S, Zaher H, Noonan BP, Burbrink FT (2013). Molecular Phylogenetics and Evolution, 66, 953-959.

Crother Bl, editor (2012) Scientific and standard English names of amphibians and reptiles of North America north of Mexico, with comments regarding confidence in our understanding, seventh edition. SSAR Herpetolog- 
ical Circular, 39, 1-92.

Daltry JC (2009) The Status and Management of Saint Lucia's Forest Reptiles and Amphibians. Technical Report No. 2 to the National Forest Demarcation and Bio-Physical Resource Inventory Project, FCG International Ltd, Helsinki, Finland.

Daltry JC, Powell R, Henderson RW (2016) Clelia errabunda (errata version published in 2017). The IUCN Red List of Threatened Species, 2016, e.T203441A115349952.

Daza JD, Bauer AM (2012) A new amber-embedded sphaerodactyl gecko from Hispaniola, with comments on morphological synapomorphies of the Sphaerodactylidae. Breviora, 529, 1-28.

Daza JD, Bauer AM, Wagner P, Böhme W (2012) A reconsideration of Sphaerodactylus dommeli Böhme, 1984 (Squamata: Gekkota: Sphaerodactylidae), a Miocene lizard in amber. Erneute Bewertung der miozänen Bernsteinechse Sphaerodactylus dommeli Böhme, 1984 (Squamata: Gekkota: Sphaerodactylidae). Journal of Zoological Systematics and Evolutionary Research, 51, 55-63.

de Queiroz K (2011) Plural versus singular common names for amphibian and reptile species. Herpetological Review, 42, 339-342.

de Queiroz K, Mayer GC (2011) Anolis roosevelti. The IUCN Red List of Threatened Species, 2011, e.T1319A3418702.

de Queiroz K, Reeder TW, Leaché A (2017) Squamata (in part) - Lizards, pages 38-58. In: Scientific and standard English names of amphibians and reptiles of North America north of Mexico, with comments regarding confidence in our understanding, eighth edition, Crother BI, editor, SSAR Herpetological Circular, 43, 1-102.

Dewynter M (2017) Pholidoscelis cineraceus (amended version of 2016 assessment). The IUCN Red List of Threatened Species, 2017, e.T1119A121639617.

Dewynter M, Powell R (2017) Pholidoscelis major (amended version of 2016 assessment). The IUCN Red List of Threatened Species, 2017, e.T1120A121642128.

Dewynter M, Daltry JC, Powell R (2016) Erythrolamprus cursor (errata version published in 2017). The IUCN Red List of Threatened Species, 2016, e.T12079A115104218.

Dutton A, Carlson AE, Long AJ, Milne GA, Clark PU, DeConto R, Horton BP, Rahmstorf S, Raymo ME (2015) Sea-level rise due to polar ice-sheet mass loss during past warm periods. Science 349, aaa4019.

Etheridge R (1964) Late Pleistocene lizards from Barbuda, British West Indies. Bulletin of the Florida Museum of Natural History, 9, 43-75.

Etheridge R (1965) Fossil lizards from the Dominican Republic. Quarterly Journal of the Florida Academy of Science, 28, 83-105.

Etheridge R (1966, "1965") Pleistocene lizards from New Providence. Quarterly Journal of the Florida Academy of Sciences, 28, 349-358.

FAO (2015) Global Forest Resources Assessment 2015. Rome: Food and Agriculture Organization of the United Nations.

Frank N, Ramus E (1995) A complete guide to scientific and common names of reptiles and amphibians of the world. $\mathrm{N}$. G. Publishing, Pottsville, Pennsylvania.

GADM (2018) Global Administrative Areas. Available online at http://www.gadm.org/ (accessed 13 November 2018). University of California, Davis.

Galis F, Arntzen JW, Lande R (2010) Dollo's Law and the irreversibility of digit loss in Bachia. Evolution, 64, 24662476.

Gehara M, Crawford AJ, Orrico VG, Rodríguez A, Lötters S, Fouquet A, Barrientos LS, Brusquetti F, De la Riva I, Ernst R, Urrutia GG, Glaw F, Guayasamin JM, Hölting M, Jansen M, Kok PJ, Kwet A, Lingnau R, Lyra M, Moravec J, Pombal JP Jr, Rojas-Runjaic FJ, Schulze A, Señaris JC, Solé M, Rodrigues MT, Twomey E, Haddad CF, Vences M, Köhler J. (2014) High levels of diversity uncovered in a widespread nominal taxon: continental phylogeography of the neotropical tree frog Dendropsophus minutus. PLoSOne, 9, e103958.

Glor RE, Laport RG (2012) Are subspecies of Anolis lizards that differ in dewlap color and pattern also genetically 
distinct? A mitochondrial analysis. Molecular Phylogeny and Evolution, 64, 255-260.

Guyer C, Savage JM (1986) Cladistic relationships among anoles (Sauria: Iguanidae). Systematic Zoology, 35, 509531.

Harvey MB, Ugueto GN, Gutberlet Jr RL (2012) Review of teiid morphology with a revised taxonomy and phylogeny of the Teiidae (Lepidosauria: Squamata). Zootaxa, 3459, 1-156.

Hedges SB (1996) Historical biogeography of West Indian vertebrates. Annual Review of Ecology and Systematics 27, 163-196.

Hedges SB (2006) Paleogeography of the Antilles and origin of West Indian terrestrial vertebrates. Annals of the Missouri Botanical Garden 93, 231-244.

Hedges SB (2018a) Caribherp: amphibians and reptiles of Caribbean Islands. Available online at http://www.caribherp.org/ (accessed 9 December 2018). Philadelphia, Pennsylvania.

Hedges SB (2018b) Caribmap: a cartographic history of the West Indies. Available online at http://www.caribmap. org/ (accessed 9 December 2018). Philadelphia, Pennsylvania.

Hedges SB, Conn CE (2012) A new skink fauna from Caribbean islands (Squamata, Scincidae, Mabuini). Zootaxa, $3228,1-244$.

Hedges SB, Wilson BS, Gibson R (2016) Hypsirhynchus ater (errata version published in 2017). The IUCN Red List of Threatened Species, 2016, e.T940A115053626.

Henderson RW, Murphy JC (2012) The Collared Tree Lizard, Plica plica (Tropiduridae, on Grenada. IRCF Reptiles \& Amphibians, 19, 126-127.

Henderson RW, Powell R (1996) Alsophis sanctaecrucis. Catalogue of American Amphibians and Reptiles, 634, 1-2.

Henderson RW, Powell R (2018) Amphibians and Reptiles of the St. Vincent and Grenada Banks, West Indies. Frankfurt, Germany: Edition Chimaira.

Hopkins DJ (1997) Merriam-Webster's Geographical Dictionary. Third edition: Merriam-Webster.

Iguana Taxonomy Working Group (2016) A checklist of the iguanas of the world (Iguanidae; Iguaninae), pages 4-46. In: Iguanas: Biology, Systematics, and Conservation, Iverson JB, Grant TD, Knapp CR, Pasachnik SA, editors. Herpetological Conservation and Biology, 11, Monograph 6.

IHO (1953) Limits of oceans and seas. Special publication No. 23. Monaco: International Hydrographic Organization.

IUCN (2018) IUCN Redlist of Threatened Species. Available from http://www.iucnredlist.org/ (accessed 8 February 2018). Gland, Switzerland: International Union for the Conservation of Nature.

John RR, Bentz EJ, Rivera Rodríguez MJ, Bauer AM, Powell R (2012) Bachia heteropa. Catalogue of American Amphibians and Reptiles, 894, 1-9.

Khan NS, Ashe E, Horton BP, Dutton A, Kopp RE, Brocard G, Engelhart SE, Hill DF, Peltier WR, Vane CH, Scatena FN (2017) Drivers of Holocene sea-level change in the Caribbean. Quaternary Science Reviews 155. 13-36.

Kohlsdorf T, Lynch VJ, Rodrigues MT, Brandley MC, Wagner GP (2010) Data and data interpretation in the study of limb evolution: A reply to Galis et al. on the revolution of digits in the lizard genus Bachia. Evolution, 64, 2477-2485.

Kraus F, Krysko KL (2017) Alien species, pages 92-102. In: Scientific and standard English names of amphibians and reptiles of North America north of Mexico, with comments regarding confidence in our understanding, eighth edition, Crother BI, editor, SSAR Herpetological Circular, 43, 1-102.

La Marca E, Azevedo-Ramos C, Coloma LA, Ron S, Hardy J (2010) Lithobates palmipes. The IUCN Red List of Threatened Species, 2010, e.T58689A11812112.

Landestoy M, Incháustegui S, Henderson RW, Hedges B (2016) Hypsirhynchus melanichnus (errata version published in 2017). The IUCN Red List of Threatened Species, 2016, e.T190581A115327313.

Liner EA (1994) Scientific and common names for the amphibians and reptiles of Mexico in English and Spanish. Nombres científicos y communes en ingles y español de los anfibios y los reptiles de México. Society for the Study of Amphibians and Reptiles Herpetological Circular, 23, vi + 113 pp. 
MacGuidan DJ, Geneva AJ, Glor RE (2017) A genomic assessment of species boundaries and hybridization in a group of highly polymorphic anoles (distichus species complex). Ecology and Evolution, 7, 3657-3671.

Maciel NM, Collevatti RG, Colli GR, Schwartz EF (2010) Late Miocene diversification and phylogenetic relationships of the huge toads in the Rhinella marina (Linnaeus, 1758) species group (Anura: Bufonidae). Molecular Phylogenetics and Evolution, 57, 787-797.

Malhotra A, Thorpe RS (1994) Parallels between island lizards suggest selection on mitochondrial DNA and morphology. Proceedings of the Royal Society of London, B, 257, 37-42.

Malhotra A, Thorpe RS (1997) Microgeographic variation in scalation of Anolis oculatus (Dominica, West Indies): A multivariate analysis. Herpetologica, 53, 49-62.

McCranie JR, Harrison A, Orellana LV (2017) Updated population and habitat comments about the reptiles of the Swan Islands, Honduras. Bulletin of the Museum of Comparative Zoology, 161, 265-284.

McCranie JR, Wilson LD, Köhler G (2005) The amphibians and reptiles of the Bay Islands and Cayos Cochinos, Honduras. Bibliomania, Salt Lake City.

Miller KG, Wright JD, Browning JV, Kulpecz A, Kominz M, Naish TR, Cramer BS, Rosenthal Y, Peltier WR, Sosdian S (2012) High tide of the warm Pliocene: Implications of global sea level for Antarctic deglaciation. Geology, 40, 407-410.

Morgan GS (2001) Patterns of extinction in West Indian bats, pages 369-407. In: Biogeography of the West Indies: Patterns and Perspectives, Woods CA, Sergile FE, editors. CRC Press, Boca Raton, Florida.

Murphy JC, Downie JR, Smith JM, Livingstone SM, Mohammed RS, Lehtinen RM, Eyre M, Sewlal J-AN, Noriega N, Casper GS, Anton T, Rutherford MG, Braswell AL, Jowers MJ (2018) A field guide to the amphibians and reptiles of Trinidad and Tobago. Trinidad and Tobago Field Naturalists' Club, Port of Spain.

Murphy JC, Jowers MJ, Lehtinen RM, Charles SP, Colli GR, Peres Jr AK, Hendry CR, Pyron RA (2016) Cryptic, sympatric diversity in tegu lizards of the Tupinambis teguixin group (Squamata, Sauria, Teiidae) and the description of three new species. PLOS ONE, 11, e0158542.

Nicholson KE, Crother Bl, Guyer C, Savage JM (2012) It is time for a new classification of anoles (Squamata: Dactyloidae). Zootaxa, 3477, 1-108.

Pinto-Sánchez NR, Calderón-Espinosa ML, Miralles A, Crawford AJ, Ramírez-Pinilla MP (2015) Molecular phylogenetics and biogeography of the Neotropical skink genus Mabuya Fitzinger (Squamata: Scincidae) with emphasis on Colombian populations. Molecular Phylogenetics and Evolution, 93, 188-211.

Platenberg R, Powell R (2016) Borikenophis sanctaecrucis (errata version published in 2017). The IUCN Red List of Threatened Species, 2016, e.T40791A115177079.

Poe S (2013) 1986 redux: New genera of anoles (Squamata: Dactyloidae) are unwarranted. Zootaxa, 3626, 295-299.

Poe S, Nieto-Montes de Oca A, Torres-Carvajal O, de Queiroz K, Velasco JA, Truett B, Gray LN, Ryan MJ, Köhler G, Ayala-Varela F, Latella I (2017) A phylogenetic, biogeographic, and taxonomic study of all extant species of Anolis (Squamata; Iguanidae). Systematic Biology, 66, 663-697.

Poinar Jr. G, Wake DB (2015) Palaeoplethodon hispaniolae gen. n., sp. n. (Amphibia: Caudata), a fossil salamander from the Caribbean. Palaeodiversity, 8, 21-29.

Powell R (1999) Herpetology of Navassa Island, West Indies. Caribbean Journal of Science, 35, 1-13.

Powell R (2018) Leiocephalus loxogrammus. Catalogue of American Amphibians and Reptiles, 915, 1-12.

Powell R, Henderson RW (2012) Island lists of West Indian amphibians and reptiles. Bulletin of the Florida Museum of Natural History, 51, 85-166.

Pregill GK (1981) Late Pleistocene herpetofaunas from Puerto Rico. University of Kansas Museum of Natural History, Miscellaneous Publication, 71, 1-72.

Pregill GK (1984) An extinct species of Leiocephalus from Haiti (Sauria: Iguanidae). Proceedings of the Biological Society of Washington, 97, 827-833.

Pregill GK (1992) Systematics of the West Indian lizard genus Leiocephalus (Squamata: Iguania: Tropiduridae). Uni- 
versity of Kansas Museum of Natural History, Miscellaneous Publication, 84, 1-69.

Reeder TW, Cole CJ, Dessauer HC (2002) Phylogenetic relationships of whiptail lizards of the genus Cnemidophorus (Squamata: Teiidae): A test of monophyly, reevaluation of karyotypic evolution, and review of hybrid origins. American Museum Novitates, 3365, 1-61.

Revell LJ, Mahler DL, Reynolds RG, Slater GJ (2015) Placing cryptic, recently extinct, or hypothesized taxa into an ultrametric phylogeny using continuous character data: A case study with the lizard Anolis roosevelti. Evolution, 69, 1027-1035.

Rieppel O (1980) Green anole in Dominican amber. Nature, 286, 486-487.

Ross FD (1998) Crocodylus rhombifer. Catalogue of American Amphibians and Reptiles, 680, 1-18.

Santos-Barrera G, Solis F, Ibáñez R, Chaves G, Bolaños F, Wilson LD, Castro F, Coloma LA, Ron SR, Acosta-Galvis A, Morales M (2008) Lithobates vaillanti. The IUCN Red List of Threatened Species, 2008, e.T58744A11835860.

Savage JM, Guyer C (1989) Infrageneric classification and species composition of the anole genera, Anolis, Ctenonotus, Dactyloa, Norops and Semiurus (Sauria: Iguanidae). Amphibia-Reptilia, 10, 105-115.

Schwartz A (1967) The Ameiva (Lacertilia, Teiidae) of Hispaniola. III. Ameiva taeniura Cope. Bulletin of the Museum of Comparative Zoology, Harvard University, 135, 345-375.

Schwartz A, Henderson R (1991) Amphibians and reptiles of the West Indies: descriptions, distributions, and natural history. University of Florida Press, Gainesville.

Schwartz A, Thomas R (1975) A check-list of West Indian amphibians and reptiles. Carnegie Museum of Natural History Special Publication, 1, 1-216.

Sibley CG, Monroe BL Jr (1990) Distribution and Taxonomy of Birds of the World. Yale University Press, New Haven, Connecticut.

Simberloff D (1995) Why do Introduced species appear to devastate islands more than mainland areas? Pacific Science, 49, 87-97.

Smith ML, Hedges SB, Buck W, Hemphill A, Inchaustegui S, Ivie M, Martina D, Maunder M, Ortega JF (2005) Caribbean islands, pages 112-118. In: Hotspots revisited: Earth's Biologically Richest and Most Endangered Terrestrial Ecoregions, Mittermeier RA, Gil PR, Hoffmann M, Pilgrim J, Brooks T, Mittermeier CG, Lamoreux J, da Fonseca GA, editors. CEMEX, Mexico City.

Solis F, Ibánez R, Jaramillo C, Fuenmayor Q, Azevedo-Ramos C, La Marca E, Coloma LA, Ron S, Hardy J, Hedges B, Ibéné B, Breuil M, Powell R (2010) Scinax ruber. The IUCN Red List of Threatened Species, 2010,.

Stephen CL, Reynoso VH, Collett WS, Hasbun CR, Breinholt JW (2013) Geographical structure and cryptic lineages within Common Green Iguanas, Iguana iguana. Journal of Biogeography, 40, 50-62.

Surget-Groba Y, Thorpe RD (2013) A likelihood framework analysis of an island radiation: phylogeography of the Lesser Antillean gecko Sphaerodactylus vincenti, in comparison with the anole Anolis roquet. Journal of Biogeography, 40, 105-116.

Thomas R (1966) A reassessment of the herpetofauna of Navassa Island. Journal of the Ohio Herpetological Society, $5,73-89$.

Thorpe RS, Surget-Groba Y, Johanss H (2010) Genetic tests for ecological and allopatric speciation in anoles on an island archipelago. PLoS Genetics, 6, e1000929.

Thorpe RS, Barlow A, Malhotra A, Surget-Groba Y (2015) Widespread parallel population adaptation to climate variation across a radiation: Implications for adaptation to climate change. Molecular Ecology, 24, 1019-1030.

Thorpe RS, Barlow A, Surget-Groba Y, Malhotra A (2018) Multilocus phylogeny, species age and biogeography of the Lesser Antillean Anoles. Molecular Phylogenetics and Evolution, 127, 682-695.

Tollis M, Boissinot S (2014) Genetic variation in the Green Anole Lizard (Anolis carolinensis) reveals island refugia and a fragmented Florida during the Quaternary. Genetica, 142, 59-72.

Tollis M, Ausubel G, Ghimire D, Boissinot S (2012) Multi-locus phylogeographic and population genetic analysis of Anolis carolinensis: Historical demography of a genomic model species. PLOS ONE, 7, e38474. 
Tucker DB, Hedges SB, Colli GR, Pyron RA, Sites Jr JW (2017) Genomic timetree and historical biogeography of Caribbean island ameiva lizards (Pholidoscelis: Teiidae). Ecology and Evolution, 7, 7080-7090.

Turtle Extinctions Working Group (2015) Turtles and tortoises of the world during the rise and global spread of humanity: First checklist and review of extinct Pleistocene and Holocene chelonians. Chelonian Research Monographs, 5, 1-66.

Turtle Taxonomy WorkingGroup [Rhodin AGJ, Iverson JB, Bour R, Fritz U, Georges A, Shaffer HB, van Dijk PP] (2017) Turtles of the World: Annotated Checklist and Atlas of Taxonomy, Synonymy, Distribution, and Conservation Status (8th Ed.). In: Conservation Biology of Freshwater Turtles and Tortoises: A Compilation Project of the IUCN/SSC Tortoise and Freshwater Turtle Specialist Group, Rhodin AGJ, Iverson JB, van Dijk PP, Saumure RA, Buhlmann KA, Pritchard PCH, Mittermeier RA, editors. Chelonian Research Monographs, 7, 1-292.

Uetz P, Hallermann J, Hošek J, editors (2018) The Reptile Database. Accessible online at http://www.reptile-database.org. (Accessed 9 December 2018).

Ugueto GN, Harvey MB (2011) Revision of Ameiva ameiva Linnaeus (Squamata: Teiidae) in Venezuela: Recognition of four species and status of introduced populations in southern Florida, USA. Herpetological Monographs, $25,113-170$.

United Nations (2018) UNDATA. A world of information. Available online at http://data.un.org/. (Accessed 10 June 2018). United Nations, New York.

Vallinoto M, Sequeira F, Sodré D, Bernardi JAR, Sampaio I, Schneider H (2010) Phylogeny and biogeography of the Rhinella marina species complex (Amphibia, Bufonidae) revisited: Implications for Neotropical diversification hypotheses. Zoologica Scripta, 39, 128-140.

van Buurt G (2004) Fieldguide to the Amphibians and Reptiles of Aruba, Curaçao and Bonaire. Frankfurt, Germany: Edition Chimaira.

Varona LS (1966) Notas sobre los crocodílidos de Cuba y descripción de una nueva especie del Pleisotoceno. Poeyana, Serie $A, 16,1-34$.

Varona LS (1984) Los cocodrilos fósiles de Cuba (Reptilia: Crocodylidae). Caribbean Journal of Science, 20, 13-18.

Watson, K. (2008) Natural history observations for 2008. Journal of the Barbados Museum Historical Society, 54, 261268.

Weiss AJ, Hedges SB (2007) Molecular phylogeny and biogeography of the Antillean geckos Phyllodactylus wirshingi, Tarentola americana, and Hemidactylus haitianus (Reptilia, Squamata). Molecular Phylogenetics and Evolution, 45, 409-416.

Williams EE (1989) Old problems and new opportunities in West Indian biogeography, pages 1-46. In: Biogeography of the West Indies: Past, Present, and Future, Woods CA, editor. Sandhill Crane Press, Gainesville, Florida.

Williams EE (1989) A critique of Guyer and Savage (1986): Cladistic relationships among anoles (Sauria: Iguanidae); Are the data available to reclassify the anoles?, pages 433-478. In: Biogeography of the West Indies: Past, Present, and Future, Woods CA, editor. Sandhill Crane Press, Gainesville, Florida.

Williamson KE, Powell R (2004) Gymnophthalmus underwoodi. Catalogue of American Amphibians and Reptiles, 793, $1-5$.

Wilson BS, Hedges SB (2016) Tarentola albertschwartzi. The IUCN Red List of Threatened Species, 2016, e. T29456A77337835.

Wilson BS, Hedges SB, World Conservation Monitoring Centre (2017) Celestus occiduus. The IUCN Red List of Threatened Species, 2017, e.T4097A71739494. 